\title{
High-content analysis and Kinetic Image Cytometry identify toxic and epigenotoxic effects of HIV antiretrovirals on human iPSC-neurons and primary neural precursor cells
}

Alyson S. Smith¹, Soneela Ankam¹, Chen Farhy², Lorenzo Fiengo², Ranor C.B. Basa', Kara L. Gordon$^{1}$, Charles T. Martin ${ }^{1}$, Alexey V. Terskikh², Kelly L. Jordan-Sciutto ${ }^{3,4}$, Jeffrey H. Price ${ }^{1,5}$, Patrick M. McDonough ${ }^{1}$

1Vala Sciences, Inc., San Diego, CA; ${ }^{2}$ Sanford Burnham Prebys Medical Discovery Institute, La Jolla, CA; ${ }^{3}$ Department of Pathology, Perelman School of Medicine, University of Pennsylvania, Philadelphia, PA; ${ }^{4}$ Department of Basic and Translational Sciences, School of Dental Medicine, University of Pennsylvania, Philadelphia, PA; ${ }^{5}$ Scintillon Institute, San Diego, CA

To whom correspondence should be addressed: asmith@valasciences.com 


\section{Abstract}

Despite viral suppression due to combination antiretroviral therapy (cART), HIV-associated neurocognitive disorders (HAND) continue to affect half of people with HIV, suggesting that certain antiretrovirals (ARVs) may contribute to HAND. We examined the effects of nucleoside/nucleotide reverse transcriptase inhibitors tenofovir disproxil fumarate (TDF) and emtricitabine (FTC) and the integrase inhibitors dolutegravir (DTG) and elvitegravir (EVG) on viability, structure, and function of glutamatergic neurons (a subtype of CNS neuron involved in cognition) derived from human induced pluripotent stem cells (hiPSC-neurons), and primary human neural precursor cells (hNPCs), which are responsible for neurogenesis. Using automated digital microscopy and image analysis (high content analysis, HCA), we found that DTG, EVG, and TDF decreased hiPSC-neuron viability, neurites, and synapses after seven days of treatment. Analysis of hiPSC-neuron calcium activity using Kinetic Image Cytometry (KIC) demonstrated that DTG and EVG also decreased the frequency and magnitude of intracellular calcium transients. Longer ARV exposures and simultaneous exposure to multiple ARVs increased the magnitude of these neurotoxic effects. Using the Microscopic Imaging of Epigenetic Landscapes (MIEL) assay, we found that TDF decreased hNPC viability and changed the distribution of histone modifications that regulate chromatin packing, suggesting that TDF may reduce neuroprogenitor pools important for CNS development and maintenance of cognition in adults. This study establishes human preclinical assays that can screen potential ARVs for CNS toxicity to develop safer CART regimens and HAND therapeutics.

Keywords: Human induced pluripotent stem cell-derived glutamatergic neurons; human neural precursor cells; HIV antiretrovirals; HIV-associated neurocognitive disorder; high content analysis; kinetic image cytometry; calcium transients; neurites; synapses; epigenetic regulation 


\section{Introduction}

About 40 million people live with human immunodeficiency virus (HIV) infection globally, and there are $\sim 1.7$ million new infections per year ${ }^{1}$. Combination antiretroviral therapy (cART), which involves simultaneous treatment with 2-4 antiretrovirals (ARVs), suppresses HIV replication, prevents progression to acquired immunodeficiency syndrome (AIDS), and extends life expectancy in adults with HIV to near normal ${ }^{2,3}$. Preexposure prophylaxis with cART can also prevent transmission of HIV to HIV-negative adolescents and adults at risk for behaviorally acquiring HIV4-8, as well as mother-to-child transmission ${ }^{9,10}$. cART has greatly reduced the incidence of AIDS-defining illnesses that affect the central nervous system (CNS), including opportunistic infections, primary CNS lymphoma, and HIV-associated dementia (HAD) ${ }^{11-14}$. However, the post-cART era has seen increased incidence of milder forms of HIV-associated neurocognitive disorder (HAND), including mild cognitive disorder (MND) and asymptomatic neurocognitive impairment $(\mathrm{ANI})^{15,16}$. HAND affects approximately $50 \%$ of people living with HIV, including patients with undetectable viral loads ${ }^{17,18}$ and can impact quality of life, employment, treatment adherence, and survival ${ }^{19-21}$. HAND can also increase the risk for progression to more severe cognitive impairment ${ }^{22,23}$.

While many factors likely contribute to cognitive impairment in people with HIV on cART, there is growing concern that $\mathrm{ARV}$ neurotoxicity contributes ${ }^{24}$. In pigtail macaques, rodents, and cultured rodent neurons, ARV exposure can cause oxidative stress, endoplasmic reticulum stress, mitochondrial dysfunction, loss of neurites and synapses, and/or neuronal cell death ${ }^{25-28}$. In people with HIV, cART can reduce neuronal metabolite levels ${ }^{29}$, the volume and structural integrity of cortical white matter ${ }^{30,31}$, and cognitive reserve as measured by functional $\mathrm{MRI}^{32}$. While some studies link cART regimens with high CNS penetration effectiveness (CPE) with improved cognition $^{33}$ or no effect on cognition ${ }^{34}$, others link high CPE with worse cognitive outcomes ${ }^{35,36}$, including a study with 61,938 people with HIV $^{37}$. Treatment interruption in HIV+ individuals with 
stable immune function can also improve neurocognitive performance for extended time periods $^{38}$. People with HIV must remain on cART for their lifetime to maintain viral suppression $^{39,40}$, leaving them vulnerable to increased neurotoxicity during neurodevelopment and aging. Indeed, children with HIV receiving cART have reduced cognition ${ }^{41-43}$, and HIVchildren who received perinatal cART experience developmental delays ${ }^{44}$. cART also increases production and reduces clearance of Alzheimer's disease-associated beta amyloid peptides in vitro $^{45-47}$ and in patients ${ }^{48,49}$, which may accelerate CNS aging and cognitive decline.

In previous research using $\mathrm{HCA}$ and $\mathrm{KIC}$, we found that tamoxifen, an anti-cancer agent linked to post-chemotherapy cognitive impairment, reduces synapses and calcium transient activity of primary rat hippocampal neurons, establishing that these in vitro techniques can test agents for potential negative impacts on cognition ${ }^{50}$. In this study, we aimed to develop human preclinical neurosafety testing platforms to screen ARVs for a broad range of potential neurotoxic and neurodevelopmental effects. We first developed HCA and KIC assays using glutamatergic neurons differentiated from human induced pluripotent stem cells (hiPSC-neurons), which provide an in vitro model system with similar gene expression, cell biology, and electrophysiology to neurons in the human brain in which to test directly for compound toxicity ${ }^{51-53}$. We also developed an assay to test $\mathrm{ARVs}$ for potential effects on neurogenesis, a process by which human neural precursor cells (hNPCs) differentiate to neurons to support central nervous system development and cognitive function in adults ${ }^{54,55}$. For this, we used the MIEL assay, a multiparametric approach to identify changes in histone acetylation and methylation patterns using $\mathrm{HCA}$ of immunofluorescence images ${ }^{56}$.

Using HCA and KIC methods on hiPSC-neurons, we found that the nucleoside/nucleotide reverse transcriptase inhibitors tenofovir disproxil fumarate (TDF) and emtricitabine (FTC) and the integrase inhibitors dolutegravir (DTG) and elvitegravir (EVG) altered key neuronal structures and functions to varying extents, and that combinations of these ARVs had additive effects. 
bioRxiv preprint doi: https://doi.org/10.1101/2020.09.05.284422; this version posted January 11,2021 . The copyright holder for this preprint (which was not certified by peer review) is the author/funder, who has granted bioRxiv a license to display the preprint in perpetuity. It is made available under aCC-BY-NC-ND 4.0 International license.

Additionally, the MIEL assay showed that TDF reduced viability and altered histone acetylation and methylation in hNPCs, indicating an epigenotoxic effect. Our findings suggest novel preclinical research strategies to test candidate $A R V s$ in vitro and to aid in identifying ARVs with reduced neurocognitive effects. 


\section{Materials and Methods}

Cell Culture. For hiPSC-neuron experiments, imaging-quality polystyrene 384-well plates (Greiner Bio-One \#781090; Frickenhausen, Germany) were coated with $0.1 \%$ polyethyleneimine and $0.028 \mathrm{mg} / \mathrm{mL}$ growth factor-reduced Matrigel (Corning Life Sciences \#354230, Tewksbury, MA, USA). hiPSC-derived glutamatergic neurons (iCell GlutaNeurons; Fujifilm CDI \#C1060, Madison, WI, USA) were seeded at a density of 60,000 live cells $/ \mathrm{cm}^{2}$ and were maintained per the manufacturer's instructions for a total of 14 days before assay.

For hNPC experiments, imaging-quality polystyrene 384-well plates (Greiner Bio-One \#781090; Frickenhausen, Germany) were coated with $0.028 \mathrm{mg} / \mathrm{mL}$ growth factor-reduced Matrigel (Corning Life Sciences \#354230, Tewksbury, MA, USA). Fetal hNPCs (Thermo Fisher Scientific \# A15654; Waltham, MA, USA) were seeded at a density of 18,200 live cells $/ \mathrm{cm}^{2}$ and were maintained in differentiation medium per the manufacturer's instructions for a total of 7 days before assay.

Test Compounds. hiPSC-neuron cultures were treated with ARVs or ARV combinations for 1 or 7 days prior to assays. The following ARVs were used alone or in combination in this study: dolutegravir (DTG; Toronto Research Chemicals \#D528800; Toronto, ON, Canada), elvitegravir (EVG; Toronto Research Chemicals \#E509000), tenofovir disproxil fumarate (TDF; Toronto Research Chemicals \#T018505), emtricitabine (FTC; Toronto Research Chemicals \#E525000), $\mathrm{DTG}+\mathrm{TDF}+\mathrm{FTC}, \mathrm{EVG}+\mathrm{TDF}+\mathrm{FTC}$, and TDF+FTC. Vehicle (0.2\% DMSO) and antagonist control treatments (25 $\mu \mathrm{M}$ para-nitroblebbistatin; Optopharma Ltd. \#DR-N-111; Budapest, Hungary) were also applied to the cells. hNPCs were treated with $0.2 \%$ DMSO, ARVs, SAHA (Cayman Chemical \#10009929; Ann Arbor, MI, USA), GSK343 (Cayman Chemical \#14094), tofacitinib (TOF, Cayman Chemical \#11598), or (+)-JQ1 (Cayman Chemical \#11187). 
Automated Microscopy. The fixed-endpoint synapse and neurite length assay and microscopic imaging of the epigenetic landscape (MIEL) assay, as well as the live-cell calcium KIC assay were imaged using the IC200-KIC automated microscope (Vala Sciences Inc.; San Diego, CA, USA; Kinetic Image Cytometry ${ }^{\circledR}$ and $\mathrm{KIC} \circledast$ are registered trademarks of Vala Sciences Inc.) outfitted with a Plan Apo 20X/0.75 NA objective lens (Nikon Instruments Inc.; Melville, NY, USA). For livecell assays, the environmental chamber of the IC200-KIC was set to $37^{\circ} \mathrm{C} / 5 \% \mathrm{CO}_{2}$.

Synapse Density and Neurite Length Assay. After treatment, cells were fixed in $2 \%$ paraformaldehyde $1.67 \%$ sucrose in HBSS without $\mathrm{Ca}^{++} / \mathrm{Mg}^{++}$, permeabilized in $0.3 \%$ Triton X100 in PBS with $\mathrm{Ca}^{++} / \mathrm{Mg}^{++}$, and blocked in 5\% normal goat serum/1\% BSA/0.1\% Triton X-100. The following primary antibodies were diluted in blocking buffer and applied to cells overnight at $4^{\circ} \mathrm{C}$ : chicken anti- $\beta$ III tubulin (Tuj-1, 1:200; Abcam \#ab41489; Boston, MA, USA), rabbit antiPSD95 (1:200; Thermo Fisher Scientific \#51-6900; Waltham, MA, USA), and mouse anti-SV2 (1:150; Developmental Studies Hybridoma Bank; lowa City, IA, USA). The next day, the following secondary antibody cocktail with Hoechst nuclear stain was made in $2 \%$ BSA and applied to the cells for 1 hour at room temperature in the dark: goat anti-chicken IgY Alexa Fluor 555 (1:500; Thermo Fisher Scientific \#A21437), goat anti-rabbit IgG Alexa Fluor 647 (1:500; Thermo Fisher Scientific \#A21245), goat anti-mouse IgG Alexa Fluor 488 (1:500; Thermo Fisher Scientific \#A11029), and $10 \mu \mathrm{g} / \mathrm{mL}$ Hoechst 33342 (Thermo Fisher Scientific \#H3570). For each well, a 3by-3 matrix of images was acquired in each optical channel.

Calcium KIC Assay. Neurons were incubated in a calcium indicator dye solution consisting of 5 $\mu \mathrm{M}$ Rhod-4 AM (AAT Bioquest \#21122; Sunnyvale, CA, USA), 1X PowerLoad (Thermo Fisher Scientific \#P10020), $1 \mu \mathrm{g} / \mathrm{mL}$ Hoechst 33342, and $2.5 \mathrm{mM}$ probenecid in phenol red-free BrainPhys (Stemcell Technologies \#05791; Vancouver, BC, Canada) for 40 min at $37^{\circ} \mathrm{C}$. The neurons were then rinsed with and imaged under phenol red-free BrainPhys. The calcium indicator dye solution and the imaging media did not contain DMSO or ARVs. One field of view 
was imaged per well, including a single image in the nuclear channel followed by a calcium movie acquired at 4 frames per second for 2 minutes.

hNPC Microscopic Imaging of Epigenetic Landscape (MIEL) Assay. After treatment, cells were fixed in $2 \%$ paraformaldehyde $/ 1.67 \%$ sucrose in $\mathrm{HBSS}$ without $\mathrm{Ca}^{++} / \mathrm{Mg}^{++}$and blocked/permeabilized in PBS with $\mathrm{Ca}^{++} / \mathrm{Mg}^{++}$and $2 \% \mathrm{BSA} / 0.5 \%$ Triton $\mathrm{X}-100$. The following primary antibodies were diluted in blocking buffer and applied to cells overnight at $4^{\circ} \mathrm{C}$ to label key histone modifications associated with epigenetic regulation: rabbit H3K9me3 (1:500; Active Motif, 39765; Carlsbad, CA, USA); mouse H3K4me1 (1:100; Active Motif, 39635; Carlsbad, CA, USA); mouse H3K27me3 (1:250; Active Motif, 61017; Carlsbad, CA, USA); and rabbit H3K27ac (1:500; Active Motif, 39133; Carlsbad, CA, USA). Two sets of cells were immunolabeled for the biomarkers: one set with H3K27me3 and H3K27ac and a second set with H3K9me3 and H3K4me1. The next day, the following secondary antibody cocktail with Hoechst nuclear stain was made in $2 \%$ BSA and applied to the cells for 1 hour at room temperature in the dark: goat anti-rabbit IgG Alexa Fluor 647 (1:500; Thermo Fisher Scientific \#A21245), goat anti-mouse IgG Alexa Fluor 488 (1:500; Thermo Fisher Scientific \#A11029), and $10 \mu \mathrm{g} / \mathrm{mL}$ Hoechst 33342 (Thermo Fisher Scientific \#H3570).

Automated Image Analysis. For hiPSC-neuron synapse density, neurite length, and calcium $\mathrm{KIC}$ assays, images were analyzed using custom algorithms in CyteSeer software (Vala Sciences Inc.; CyteSeer® is a registered trademark of Vala Sciences Inc.). The NO2 v7.2.4 Neurite Morphology and Synapse Density with Somas algorithm was used to analyze the synapse density and neurite length images. This algorithm identified live neuronal nuclei as relatively large nuclei with diffuse Hoechst staining (as opposed to small, bright, dead nuclei) that colocalize with neuron-specific $\beta$ III-tubulin (Tuj-1) staining. The algorithm then identified Tuj-1+ neurites, which includes axons and dendrites. Small neurite fragments and Tuj-1+ cellular debris were excluded, as well as the Tuj-1 positive neuronal cell bodies (somas). To identify synapses, the algorithm 
first identified SV2+ (presynaptic) and PSD95+ (postsynaptic) puncta with areas between 0.32 and $1.27 \mu \mathrm{m}^{2}$ (between 3 and 15 pixels $^{2}$ with a pixel size of $0.325 \mu \mathrm{m}$ ). To be considered synapses, the SV2+ puncta centroids were required to be within $1.95 \mu \mathrm{m}$ (6 pixels) of the nearest neurite and within $0.975 \mu \mathrm{m}$ (3 pixels) of the nearest PSD95+ puncta centroid. Neuronal viability, neurite, and synapse data are presented as one data point per well, with each data point representing the average value across each of the 9 fields of view acquired per well. Each field of view contained about 200 live neurons in DMSO-treated control wells.

The calcium KIC assays were analyzed with the Neuron Calcium KIC v10.5 algorithm. Neuronal cell bodies were identified as Rhod-4 AM-positive areas associated with live neuron nuclei. The average pixel intensity of Rhod-4 AM signal in each cell body was measured for each of the 480 frames (4 frames per second for two minutes). The resulting functions of Rhod-4 AM average pixel intensity over time were baseline subtracted to determine if each neuron displayed calcium transients during the recording period and to calculate the event frequencies and mean peak amplitudes for each active cell. Event frequency and mean peak amplitude data are presented as one data point per well, with each data point representing the average value of all active neurons in each field of view. Each field of view contained about 200 live neurons in DMSOtreated control wells.

For the MIEL assay, images were analyzed using Acapella 2.6 (PerkinElmer), and image texture features were analyzed as described in Farhy, et al., eLife, 201956. Quadratic discriminant analysis was performed on histone modification texture features using the Excel add-on program XIstat (Base, v19.06).

Statistical Analysis. The data were analyzed with Prism (GraphPad Software; San Diego, CA, USA) using ANOVA, followed by either Dunnett's or Tukey's post-hoc multiple comparisons test for statistical significance $\left({ }^{*} p<0.05,{ }^{* *} p<0.01,{ }^{* *} p<0.001\right)$. 


\section{Results}

\section{HCA to characterize HIV ARV effects on neuronal viability and neurites in hiPSC-neurons.}

To test for ARV neurotoxicity, we exposed hiPSC-neurons to $0.1,1$, or $10 \mu \mathrm{M}$ of DTG, EVG, TDF, or FTC for seven days. We chose these concentrations to test for neurotoxic effects at levels below, near, and above the plasma Cmax of each ARV (Table 1). We used $25 \mu \mathrm{M}$ blebbistatin, a nonmuscle myosin II inhibitor ${ }^{57,58}$, as a control compound. We then fixed the hiPSC-neurons and immunolabeled for Tuj-1 (neuron-specific ßlll-tubulin), SV2 (presynaptic protein), PSD95 (postsynaptic protein), stained with Hoechst (nuclei), and imaged the cells with the IC200 Image Cytometer to assess neuronal viability and morphology. Images of Tuj-1 and nuclei show significant loss of neurites in hiPSC-neurons treated with $10 \mu \mathrm{M} E V G$, indicating a neurotoxic effect, with smaller effects for the other ARVs (Fig. 1A). Image analysis with a CyteSeer algorithm that identifies the nuclei corresponding to live neurons (see Methods) determined that Neuronal

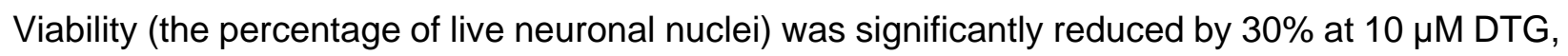
$65 \%$ at $10 \mu \mathrm{M}$ EVG, and 35\% at $10 \mu \mathrm{M}$ TDF (Fig. 1B). We did not observe changes in Neuronal Viability at lower concentrations of TDG, EVG, or TDF, or at any tested concentration of FTC.

To further quantify effects of the ARVs, we used a CyteSeer image analysis algorithm that traces Tuj-1-positive neurites and calculates their length. The total neurite length per $\mathrm{cm}^{2}$ image area (Total Neurite Length) was significantly reduced by $20 \%$ at $10 \mu \mathrm{M}$ DTG, $80 \%$ at $10 \mu \mathrm{M} \mathrm{EVG}$, and $20 \%$ at $10 \mu \mathrm{M}$ TDF (Fig. 1C). The Neurite Length per Neuron (Total Neurite Length divided by the number of live neuronal nuclei) was slightly but significantly increased by $20 \%(p<0.05)$ after treatment with $10 \mu \mathrm{M}$ DTG but was $50 \%$ lower after treatment with $10 \mu \mathrm{M}$ EVG (Fig. 1D). The Total Neurite Length and Neurite Length per Neuron remained similar to DMSO alone at lower concentrations of DTG, EVG, and TDF, and at all tested concentrations of FTC. 
Most HIV infections are treated with combination antiretroviral therapy (cART) consisting of two, three, or four ARVs from two or more different classes ${ }^{2-4}$. To test whether cART causes increased neurotoxicity and neurite loss compared to single ARVs, we compared Neuronal Viability and neurite length measurements in hiPSC-neurons treated with $10 \mu \mathrm{M} D T G, E V G, T D F$, FTC, or three combinations (DTG/TDF/FTC, EVG/TDF/FTC, or TDF/FTC; all ARVs at $10 \mu M)$. DTG/TDF/FTC has been the primary cART regimen in use in Botswana since 2016 for all people with HIV, including pregnant women ${ }^{59}$. TDF/FTC and EVG/TDF/FTC are components of Truvada and Stribild, respectively (made by Gilead Sciences, Stribild also features cobicistat).

Treatment with single or combination ARVs for one day did not affect Neuronal Viability, Total Neurite Length, or Neurite Length per Neuron (Fig. 2A-C), although blebbistatin increased Total Neurite Length by 15\% (Fig. 2B). By contrast, seven-day ARV and cART exposure caused significant neurotoxicity. Neuronal Viability was significantly reduced by $20 \%$ by $10 \mu \mathrm{M}$ DTG, $80 \%$ by $10 \mu \mathrm{M}$ EVG, $30 \%$ by $10 \mu \mathrm{M}$ TDF, $70 \%$ by DTG/TDF/FTC, $85 \%$ by EVG/TDF/FTC, and $30 \%$ by TDF/FTC (Fig. 2D). We used the Tukey's multiple comparisons test to test for significant differences between data from all test conditions and the DMSO control and between single ARVs to the ARV combinations (see Supplemental Data for full results). Comparing ARV combinations to their components, TDF/FTC treatment led to similar Neuronal Viability to that of TDF alone, suggesting FTC does not affect TDF toxicity (Table S1). EVG/TDF/FTC treatment also led to similar Neuronal Viability to that of EVG alone, suggesting that TDF and FTC do not affect EVG toxicity. By contrast, DTG/TDF/FTC treatment significantly reduced Neuronal Viability compared to either DTG alone or TDF/FTC ( $p<0.001$ for both comparisons), suggesting additive toxicity by these ARVs.

Seven-day ARV exposure also significantly reduced Total Neurite Length for hiPSCneurons treated with $10 \mu \mathrm{M}$ EVG (85\% reduction), DTG/TDF/FTC (55\%), and EVG/TDF/FTC (90\%) (Fig. 2E). DTG/FTC/TDF treatment led to a 50\% lower Total Neurite Length than DTG 
alone $(p<0.001)$, while EVG/TDF/FTC treatment led to a similar neurite length to that of EVG alone. Neurite lengths were similar to DMSO for hiPSC-neurons treated with TDF, FTC, or TDF/FTC. Neurite Length per Neuron did not change significantly with any ARV treatment, but there was increased variability in wells treated with EVG, DTG/TDF/FTC, and EVG/TDF/FTC (Fig. 2F). See Tables S2 and S3 for the results of comparisons between each condition for Total Neurite Length and Neurite Length per Neuron.

For both Neuronal Viability and Total Neurite Length, EVG displayed dominant neurotoxic effects in combination treatments (EVG alone reduced Neuronal Viability and Total Neurite Length to the same degree as EVG/TDF/FTC), while DTG and TDF had additive effects (DTG/TDF/FTC reduced Neuronal Viability and Total Neurite Length to a greater extent than either DTG or TDF/FTC).

HCA to characterize HIV ARV effects on synapses in hiPSC-neurons. To measure the effects of ARV treatments on hiPSC-neuron synapses, we used a CyteSeer image analysis algorithm that identifies synapses as SV2 (presynaptic) and PSD95 (postsynaptic) puncta near each other and Tuj-1-positive neurites. We defined Synapse Density as the number of synapses per $\mathrm{cm}^{2}$ of the imaging area and Synapses/Neurite Length as the number of synapses per Total Neurite Length (in microns). Figure 3A shows representative images of SV2 (left) and PSD95 (center) staining of hiPSC-neurons exposed to ARVs for seven days and the live neuronal nuclei (green), neurites (cyan), and synapses (magenta) identified by CyteSeer (right). hiPSC-neurons treated with $10 \mu \mathrm{M}$ EVG had fewer synapses, with smaller effects for the other ARVs. Effects on hiPSCneuron synapses appeared at lower doses and for more ARVs than the effects on neuronal viability and neurite length. Synapse Density was significantly reduced by $1 \mu$ M DTG (30\%), 0.1 $\mu \mathrm{M}(20 \%)$ and $10 \mu \mathrm{M}(75 \%) \mathrm{EVG}, 1 \mu \mathrm{M}(30 \%)$ and $10 \mu \mathrm{M}(40 \%)$ TDF, $1 \mu \mathrm{M}(20 \%)$ and $10 \mu \mathrm{M}$ (30\%) FTC, and blebbistatin (30\%) (Fig. 3B). DTG significantly reduced the Synapses/Neurite Length by $20 \%$ at $0.1 \mu \mathrm{M}$ and $30 \%$ at $1 \mu \mathrm{M}$ but had no effect at $10 \mu \mathrm{M}$ (Fig. 3C). EVG decreased 
the Synapses/Neurite Length by $25 \%$ at $0.1 \mu \mathrm{M}$, had no effect at $1 \mu \mathrm{M}$, and increased the Synapses/Neurite Length by $20 \%$ at $10 \mu \mathrm{M}$. TDF and FTC reduced the Synapses/Neurite Length at all tested concentrations (20\%, 25\%, and $20 \%$ for $0.1,1$, and $10 \mu \mathrm{M}$ TDF and $20 \%, 20 \%$, and $30 \%$ for $0.1,1$, and $10 \mu \mathrm{M}$ FTC). Blebbistatin reduced the Synapses/Neurite Length by $20 \%$.

In experiments comparing $10 \mu \mathrm{M}$ doses of single ARVs to ARV combinations, one-day treatments did not affect the Synapse Density or Synapses/Neurite Length, although blebbistatin increased the Synapse Density by $20 \%$ (Fig 4A, B). By contrast, the Synapse Density was significantly reduced by seven-day treatment with EVG $(90 \%)$, DTG/TDF/FTC (60\%), EVG/TDF/FTC (90\%), and TDF/FTC (20\%) (Fig. 4C). DTG/FTC/TDF treatment led to a 50\% lower Synapse Density than that of DTG alone ( $p<0.001$, Tukey's), while EVG/TDF/FTC treatment led to a similar Synapse Density to that of EVG alone. TDF/FTC treatment reduced the Synapses/Neurite Length by $20 \%$ (vs. DMSO, $p<0.001$ ), but this parameter was not affected by other ARV treatments (Fig. 4D). See Tables S4 and S5 for the results of comparisons between each condition for Synapse Density and Synapses/Neurite Length.

\section{KIC to characterize HIV ARV effects on intracellular calcium transients in hiPSC-neurons.}

Neurons exhibit action potential-dependent and -independent peaks in intracellular calcium concentration that induce molecular and structural changes within neurons through calciumsensitive effectors ${ }^{60-62}$. Dysregulation of neuronal intracellular calcium concentration and signaling occurs in aging, traumatic brain injury, and neurodegenerative diseases ${ }^{63}$. To test ARVs for effects on neuronal calcium transients, we treated hiPSC-neurons with $10 \mu \mathrm{M}$ of each ARV alone or in combination for one or seven days. We then loaded the hiPSC-neurons with Hoechst and the calcium indicator Rhod-4 and assayed the cells for calcium activity. For each well, we collected a single image in the nuclear channel and a digital movie in the Rhod-4 channel at four frames per second for two minutes. We then used a CyteSeer KIC analysis algorithm originally developed for quantifying calcium transients in cardiac myocytes ${ }^{64}$ and adapted to quantify neuronal calcium 
transients ${ }^{50}$. The algorithm measured the Rhod-4 signal in the soma associated with each live neuron nucleus at each frame, enabling detection of each calcium transient that occurred in each neuron during the recording period. The algorithm then calculated parameters including the percent of neurons with calcium transients and frequency and amplitude of the transients.

hiPSC-neurons exposed to DMSO alone for 7 days displayed between 0 and 50 calcium transients that were typically 5 to 30 seconds in duration during the two-minute recording periods (Fig. 5A). hiPSC-neurons exposed to TDF, FTC, and TDF/FTC for 7 days displayed similar calcium transient activity to those treated with DMSO alone (Fig. 5D, E, H), while neurons treated with DTG or DTG/TDF/FTC had less calcium transient activity compared to DMSO (Fig. 5B, F) and neurons treated with EVG or EVG/TDF/FTC were virtually inactive (Fig. 5C, G).

Quantification of the calcium transient activity showed no significant effects after one day of ARV exposure (Fig. 6A-C). After seven days of ARV exposure, the percent active hiPSCneurons was reduced by $30 \%$ by DTG, $90 \%$ by EVG, $70 \%$ by DTG/TDF/FTC, $95 \%$ by EVG/TDF/FTC, and $25 \%$ by TDF/FTC (Fig. 6D). DTG/TDF/FTC treatment led to $60 \%$ lower percent activity than DTG alone $(p<0.001)$, while EVG/TDF/FTC treatment led to a similar effect on percent activity to that of EVG alone. The event frequency (Fig. 6E) was significantly reduced by EVG (40\%) and by EVG/TDF/FTC (55\%), but not by any of the other treatments (while event frequency for DTG/TDF/FTC was lower than for DMSO, this difference did not achieve statistical significance). The mean peak amplitude was also significantly reduced by $10 \mu \mathrm{M}$ EVG (55\%) and EVG/TDF/FTC (50\%), but not by any of the other treatments. See Tables S6-8 for the results of comparisons between each condition for percent activity, event frequency, and mean peak amplitude.

Microscopic imaging of the epigenetic landscape (MIEL) analysis to characterize HIV ARV effects on hNPC epigenetic signature. To test for ARV effects on neurogenesis, we treated hNPCs with $0.1,1.0$, or $10 \mu \mathrm{M}$ of each ARV for three days. After treatment with $0.1,1.0$, or $10 \mu \mathrm{M}$ 
DTG, EVG, or FTC, hNPC viability was similar to DMSO controls. Treatment with $10 \mu \mathrm{M}$ TDF, however, significantly reduced hNPC viability by $30 \%$ (Fig. 7A). hNPC viability was also reduced by $3 \mu \mathrm{M}$ SAHA (45\%), a histone deacetylase inhibitor, and by $10 \mu \mathrm{M}$ GSK343 (50\%), a histone methyltransferase inhibitor. hNPC viability was not affected by $0.3 \mu \mathrm{M}$ JQ1, which inhibits the interaction of bromodomain-containing proteins with acetylated histones, or by $1 \mu \mathrm{M}$ tofacitinib, which inhibits JAK activity.

To analyze ARV effects on hNPC epigenetics, we immunolabeled hNPCs for two sets of histone modifications: H3K27me3 (condensed chromatin) and H3K27ac (active enhancers) or H3K9me3 (condensed chromatin) and H3K4me1 (primed enhancers). We then scanned the cells using Vala's IC200 Image Cytometer and analyzed the images to identify the multivariate epigenetic signatures of each histone modification in each condition. We characterized the epigenetic signatures using texture features ${ }^{56,65,66}$ rather than intensities and morphologies to reduce culturing and immunolabeling artifacts. Quadratic discriminant analysis reports changes in the epigenetic signatures in response to each treatment. SAHA and GSK43, which decreased hNPC viability, also significantly affected the H3K27me3/H3K27ac (Fig. 7B) and the H3K9me3/H3K4me1 (Fig. 7C) epigenetic signatures, with discriminant analysis separating samples treated with these compounds from DMSO- and ARV-treated samples. Of the ARVs tested, only $10 \mu \mathrm{M}$ TDF altered the epigenetic signature, with TDF-treated samples clustering together and separately from DMSO-, SAHA-, and GSK343-treated samples in both H3K27me3/H3K27ac and H3K9me3/HeK4me1 plots (Fig. 7B, C).

Confusion matrices report the ability of the multiparametric discriminant analysis to correctly classify each sample with other samples that received the same treatment compared to other treatments (Fig. 7D, E) ${ }^{56}$. The confusion matrix for both H3K27me3/H3K27ac and H3K9me3/H3K4me1 show that classification occurred correctly $100 \%$ of the time for samples treated with $10 \mu \mathrm{M}$ GSK343, $3 \mu \mathrm{M}$ SAHA, and $10 \mu \mathrm{M}$ TDF. This classification accuracy supports 
our conclusion that these three compounds are epigenotoxic and significantly alter the hNPC epigenetic landscape. Samples treated with DMSO, DTG, EVG, FTC, JQ1, or TOF, which clustered together after discriminant analysis, were correctly classified less than $100 \%$ of the time.

The results from the MIEL assay demonstrate that TDF, but not DTG, EVG, or FTC, reduced viability and altered the pattern of the H3K27me3, H3K27ac, H3K9me3, and H3K4me1 epigenetic histone modifications in hNPCs. 


\section{Discussion}

In this study, we developed $\mathrm{HCA}$ and $\mathrm{KIC}$ methods to quantify neurotoxic and neurodevelopmental effects of HIV ARVs in hiPSC-neurons and human neural precursor cells (Fig. 8). Loss of function and/or cell death in excitatory neurons resulting from ARV neurotoxicity could contribute to HIV-associated neurocognitive disorders (HAND), which currently affect about half of people with HIV ${ }^{17,18}$. Because people with HIV develop non-AIDs-related health conditions, including cognitive decline, at an earlier age than the general population ${ }^{67-69}$, HAND is likely to increase in incidence and severity as the percentage of people with HIV over 50 years old increases ${ }^{70}$. ARV exposure during fetal development, childhood, and adolescence may also increase HAND incidence by affecting neurogenesis at key stages in CNS development. Human in vitro systems are needed to identify and mitigate ARV neurotoxicity and neurodevelopmental effects. The methods used in this study feature automated digital microscopy and analysis for cell plated in 384-well dishes, a format that facilitates higher throughput testing of ARVs at multiple concentrations and combinations, as well as testing of potential HAND therapeutics that may mitigate ARV neurotoxicity.

Many previous in vitro studies of ARV neurotoxicity have been conducted with primary embryonic rat neurons. Exposure of these cells to $10 \mu \mathrm{M}$ of the integrase inhibitor EVG for four days, but not two days, significantly reduced the number of MAP2+ neurons ${ }^{28}$, while 0.1 and 1 $\mu \mathrm{M}$ EVG were not neurotoxic. In this study, we found that seven-day exposure (but not one-day exposure) to $10 \mu \mathrm{M}$ EVG reduced hiPSC-neuron viability, decreased neurite length and synapse density, and inhibited intracellular calcium transients, confirming the neurotoxicity of this compound in a human neuron model. We also observed milder toxic effects after treatment with $10 \mu \mathrm{M}$ of the integrase inhibitor DTG and $10 \mu \mathrm{M}$ of the nucleoside/nucleotide reverse transcriptase inhibitor TDF. The nucleoside/nucleotide reverse transcriptase inhibitor FTC, which forms a base 
for front-line recommended cART regimens in the US, was relatively non-toxic in our hiPSCneuron model.

Our data suggest that plasma, CSF, and brain concentrations of DTG, EVG, and TDF need to be managed to control HIV replication while minimizing ARV neurotoxicity. Efforts to increase the CNS penetration effectiveness (CPE) of ARVs such as EVG ${ }^{71}$ and protease inhibitors $^{72}$ may decrease the CNS viral load but may also increase ARV neurotoxicity. Neuroimaging studies have shown that HAND patients on cART have locally compromised blood brain barriers ${ }^{73}$, which could increase ARV levels in the brain, even for ARVs with low CPE scores. In postmortem brains from HIV-positive people who died while on cART, higher ARV levels were associated with worse antemortem cognitive performance ${ }^{74}$. This association could result from HIV-induced systemic inflammation that leads to blood brain barrier compromise or from increased ARV neurotoxicity. Our human in vitro assays enable direct testing of ARVs for neurotoxicity, which is likely to be relevant to HAND pathology.

While our assay used hiPSC-neurons in isolation, neurons coexist with glial cells like microglia and astrocytes in vivo, which impact their survival and function ${ }^{75}$ and play key roles in neuroinflammation and neurodegenerative diseases such as Alzheimer's ${ }^{76,77}$. A recently developed in vitro model enables testing for ARV neurotoxicity on co-cultured hiPSC-neurons, microglia, and -astrocytes ${ }^{78}$ in the presence or absence of HIV infection. In this system, efavirenz, a nucleoside/nucleotide reverse transcriptase inhibitor linked to neuropsychiatric and cognitive effects $^{79-81}$, increased inflammation and reduced phagocytosis in the hiPSC-microglia. Our hiPSCneuron assay could be similarly expanded to include hiPSC-microglia and -astrocytes to test whether glia contribute to or protect against ARV-induced neuronal toxicity. This expanded system will also enable testing of the efficacy of ARVs to inhibit HIV infection of human microglia and the resulting inflammation ${ }^{82,83}$. 
Our assay of ARV effects in hNPCs indicated that TDF reduces viability in this cell type. TDF and zidovidine (AZT) reduce the viability of murine NPCs ${ }^{84,85}$, but to our knowledge this is the first report of ARV effects on human NPCs. TDF also altered the distribution of histone modifications in hNPCs, which may indicate that TDF affects hNPC differentiation. Because ARVs are prescribed as combinations of several drugs, it will be important to investigate such combinations for their epigenotoxic activity. DNA methylation, another epigenetic modification, has recently been reported to be altered in $\mathrm{HIV}_{+}$children receiving $\mathrm{CART}^{86}$. Given the importance of neurogenesis in neurodevelopment and maintenance of cognitive function during aging, it is critical to evaluate existing and candidate ARVs for effects on hNPC viability and epigenetics.

In summary, we have developed and applied methods for the high throughput testing of HIV ARVs on hiPSC-neurons that model excitatory neurons of the human brain. Similar HCA and KIC methods have been applied to quantify neurotoxic effects of the breast cancer therapeutic tamoxifen in primary rat hippocampal neurons ${ }^{50}$, suggesting a broad applicability for these methods for neurotoxicity screening. We have also developed methods to screen for ARV effects on neurogenesis by quantifying the viability and epigenetics of hNPCs. Developing cART regimens that balance the neuroprotective effects of suppressing CNS HIV replication with potential ARV neurotoxicity remains a major challenge in HIV/AIDS treatment and drug discovery ${ }^{87-89}$. The results of the present study represent progress in this direction. Future studies will incorporate human glial cells (hiPSC-microglia and hiPSC-astrocytes) into our assay system to increase its power to detect neurotoxic and neurodevelopmental effects of existing and candidate anti-HIV therapeutics and to identify potential HAND therapeutics that can mitigate these effects. 


\section{Acknowledgements:}

This study was funded, in part, by grants from the NIH, which include R44ES026268 "Assay of chemicals for Parkinson's toxicity in human iPSC-derived neurons" and R41MH119621 “The Microscopic Imaging of Epigenetic Landscape- NeuroDevelopment (MIEL-ND) assay" and 1R43AG062012-01 “The Alzheimer's Therapeutics Screening Assay: a high-throughput drugdiscovery platform utilizing neurons and microglia derived from human induced pluripotent stem cells and Kinetic Image Cytometry". 


\section{Figure Legends}

Figure 1. Single ARVs decrease viability and neurite length in hiPSC-neurons in a dosedependent manner. hiPSC-neurons were exposed to the ARVs for 7 days. (A) Representative images of hiPSC-neurons treated with DMSO alone or $10 \mu \mathrm{M}$ dolutegravir (DTG), elvitegravir (EVG), tenofovir disproxil fumarate (TDF), or emtricitabine (FTC), fixed, and stained for nuclei (Hoechst, blue) and neuronal somas and neurites (Tuj-1, grayscale). Scale bar $=50 \mu \mathrm{m}$. (B-D) Viability and neurite data for hiPSC-neurons treated with DMSO alone, ARVs $(0.1,1$, or $10 \mu \mathrm{M})$ or blebbistatin $(25 \mu \mathrm{M})$. (B) Neuronal viability as defined by the number of live neuron nuclei in each well relative to the DMSO control mean. (C) Total neurite length in $\mu \mathrm{m}$ per $\mathrm{cm}^{2}$ image area for each condition. (D) Total neurite length per live neuron nucleus for each condition. Each dot represents the average of each measurement from nine images per well. DMSO and blebbistatin: $\mathrm{n}=18$ wells; ARVs: $\mathrm{n}=6$ wells. Bars represent mean \pm standard deviation. Statistics performed with one-way ANOVA followed by Dunnett's multiple comparisons test. ${ }^{*} p<0.05,{ }^{* *} p<0.01$, ${ }^{* * *}$ $\mathrm{p}<0.001$

Figure 2. Single and combined ARVs decrease viability and neurite length in hiPSCneurons after seven days, but not one day of exposure. hiPSC-neurons were treated for one (left) or seven days (right) with DMSO alone, $25 \mu \mathrm{M}$ blebbistatin, single ARVs, or combinations of ARVs (each at $10 \mu \mathrm{M})$. (A, D) Neuronal viability as defined by the number of live neuron nuclei in each well relative to the DMSO control mean. $(B, E)$ Total neurite length in $\mu \mathrm{m}$ per $\mathrm{cm}^{2}$ image area in each well relative to the DMSO control mean. (C, F) Total neurite length per live neuron nucleus in each well relative to the DMSO control mean. Each dot represents the average of each measurement from nine images per well. One-day treatment DMSO and blebbistatin: $\mathrm{n}=18$ wells; 
ARVs: $n=6$ wells. Seven-day treatment DMSO and blebbistatin: $n=36$ wells; ARVs: $n=12$ wells from two experiments. Bars represent mean \pm standard deviation. Statistics performed with oneway ANOVA followed by Tukey's multiple comparisons test. ${ }^{*} p<0.05$, ${ }^{* *} p<0.01,{ }^{* * *} p<0.001$. Significant differences between DMSO and ARV or blebbistatin treatments are indicated on each graph. Significant differences between DTG and other ARVs/combinations are indicated with "a", significant differences between EVG and other ARVs/combinations are indicated with "b", and significant differences between TDF and other ARVs/combinations are indicated with "c". Results of other Tukey's comparisons are reported in Figures S1-S3.

Figure 3. Single ARVs affect synapse density in hiPSC-neurons in a dose-dependent manner. (A) Representative images of hiPSC-neurons from the same experiment in Figure 1 (seven-day ARV treatment) stained for SV2 (presynaptic marker, left) and PSD95 (postsynaptic marker, center). Right images show live neuron nuclei (green), neurites (cyan), and synapses (magenta) identified by CyteSeer. Scale bar $=50 \mu \mathrm{m}$. (B) Synapse Density (synapses per $\mathrm{cm}^{2}$ imaging area) for each condition. (C) Synapses/Neurite Length (synapses per $\mu$ m neurite length) for each condition. Each dot represents the average of each measurement from nine images per well. DMSO and blebbistatin: $n=18$ wells; ARVs: $n=6$ wells. Bars represent mean \pm standard deviation. Statistics performed with one-way ANOVA followed by Dunnett's multiple comparisons test. ${ }^{*} p<0.05,{ }^{* *} p<0.01,{ }^{* *} p<0.001$.

Figure 4. Single and combined ARVs affect synapse density of hiPSC-neurons after seven days, but not one day of exposure. hiPSC-neurons were treated for one or seven days with DMSO alone, $25 \mu \mathrm{M}$ blebbistatin, single ARVs, or combinations of ARVs (each at $10 \mu \mathrm{M})$. (A, C) Synapse Density (synapses per $\mathrm{cm}^{2}$ image area) in each well relative to the DMSO control mean. 
(B, D) Synapses/Neurite Length (synapses per $\mu \mathrm{m}$ neurite length) in each well relative to the DMSO control mean. Each dot represents the average of each measurement from nine images per well. One day treatment DMSO and blebbistatin: $n=18$ wells; ARVs: $n=6$ wells. Seven day treatment DMSO and blebbistatin: $n=36$ wells; ARVs: $n=12$ wells from two experiments. Bars represent mean \pm standard deviation. Statistics performed with one-way ANOVA followed by Tukey's multiple comparisons test. ${ }^{*} p<0.05,{ }^{* *} p<0.01,{ }^{* * *} p<0.001$. Significant differences between DMSO and ARV or blebbistatin treatments are indicated on each graph. Significant differences between DTG and other ARVs/combinations are indicated with "a", significant differences between EVG and other ARVs/combinations are indicated with "b", and significant differences between TDF and other ARVs/combinations are indicated with "c". Results of other Tukey's comparisons are reported in Figures S4 and S5.

Figure 5. Effect of ARVs on calcium transients in hiPSC-neurons. hiPSC-neurons were treated for seven days with DMSO alone, single ARVs, or combinations of ARVs (each at $10 \mu M)$. (A-H) Traces showing transient increases in calcium fluorescence relative to baseline. Each graph contains one trace for each active neuron in each condition with detectable increases in calcium fluorescence. DMSO: 1034 cells; DTG: 716 cells; EVG: 31 cells; TDF: 729 cells; FTC: 1003 cells; DTG/TDF/FTC: 134 cells; EVG/TDF/FTC: 17 cells; TDF/FTC: 539 cells. Six wells per condition.

Figure 6. Quantification of the effects of ARVs on calcium activity in hiPSC-neurons. hiPSC-neurons were treated for one or seven days with DMSO alone, single ARVs, or combinations of ARVs (each at $10 \mu \mathrm{M})$. (A, D) Percent of live neurons that are active with detectable calcium transients in each condition. (B, E) The mean event frequency of calcium transients of all active neurons in each well. (C, F) The mean of mean calcium peak amplitudes 
of all active neurons in each well. Six wells per condition. Bars represent mean \pm standard deviation. Statistics performed with one-way ANOVA followed by Tukey's multiple comparisons test. ${ }^{*} p<0.05,{ }^{* *} p<0.01,{ }^{* *} p<0.001$ vs. DMSO. Significant differences between DTG and other ARVs/combinations are indicated with "a", significant differences between EVG and other ARVs/combinations are indicated with "b", and significant differences between TDF and other ARVs/combinations are indicated with "c". Results of other Tukey's comparisons are reported in Figures S6-S8.

Figure 7. TDF affects the viability and epigenetic signature of hNPCs. Fetal hNPCs were treated with DMSO alone, compounds with known epigenetic effects (SAHA, GSK343, JQ1, or TOF), or ARVs at the indicated concentrations. (A) Count of hNPCs per well for each condition following treatment. Bars represent mean \pm standard deviation. Statistics performed with one-way ANOVA followed by Dunnett's multiple comparisons test. * $p<0.05$. (B-E) Quadratic discriminant analysis using texture features derived from images of hNPCs treated with the indicated compounds and immunostained for H3K27me3 and H3K27ac (B, D) or H3K9me3 and H3K4me1 $(C, E) .(B, C)$ Scatter plots showing the first two texture-derived discriminant factors for each condition. (D, E) Confusion matrices showing the results of the discriminant analysis. Numbers represent the percent of wells classified correctly (on the diagonal) and incorrectly (off the diagonal). $\mathrm{N}=6$ wells per condition.

Figure 8. HCA, KIC, and MIEL to identify diverse neurotoxic effects of HIV antiretrovirals. (Top) Outline of our methods to identify HIV antiretroviral effects in hiPSC-neurons. After culture in imaging-quality 384-well plates, hiPSC-neurons are exposed to ARVs alone or in combination for one or seven days. hiPSC-neurons are then fixed and stained for high content analysis or 
imaged live for KIC of intracellular calcium transients. These assays test if ARVs alter hiPSCneuron viability, neurite length, synapse density, and/or calcium transient activity. (Bottom) Outline of our methods to identify ARV effects in hNPCs. After culture in imaging-quality 384-well plates, hNPCs are exposed to ARVs for three days. hNPCs are then fixed and stained for microscopic imaging of the epigenetic landscape (MIEL) analysis. This assay tests if ARVs affect hNPC viability and/or change the histone modification pattern within the nuclei. Figure made with Biorender.com.

Table S1. Tukey's multiple comparison test results for Neuronal Viability. Tables show the results of each pairwise comparison for hiPSC-neurons treated for one (left) or seven days (right) with DMSO alone, $25 \mu \mathrm{M}$ blebbistatin, single ARVs, or combinations of ARVs (each at $10 \mu \mathrm{M}$ ). See Figure 2A, D for graph of results. ns: not significant $(p>0.05){ }^{*} p<0.05,{ }^{\star *} p<0.01,{ }^{* \star} p<$ 0.001 .

Table S2. Tukey's multiple comparison test results for Total Neurite Length. Tables show the results of each pairwise comparison for hiPSC-neurons treated for one (left) or seven days (right) with DMSO alone, $25 \mu \mathrm{M}$ blebbistatin, single ARVs, or combinations of ARVs (each at 10 $\mu M)$. See Figure $2 B$, E for graph of results. ns: not significant $(p>0.05){ }^{*} p<0.05,{ }^{* *} p<0.01,{ }^{* * *}$ $\mathrm{p}<0.001$.

Table S3. Tukey's multiple comparison test results for Neurite Length per Neuron. Tables show the results of each pairwise comparison for hiPSC-neurons treated for one (left) or seven days (right) with DMSO alone, $25 \mu \mathrm{M}$ blebbistatin, single ARVs, or combinations of ARVs (each 
at $10 \mu \mathrm{M})$. See Figure $2 \mathrm{C}, \mathrm{F}$ for graph of results. ns: not significant $(p>0.05){ }^{*} p<0.05,{ }^{* *} p<$ $0.01,{ }^{* * *} p<0.001$

Table S4. Tukey's multiple comparison test results for Synapse Density. Tables show the results of each pairwise comparison for hiPSC-neurons treated for one (left) or seven days (right) with DMSO alone, $25 \mu \mathrm{M}$ blebbistatin, single ARVs, or combinations of ARVs (each at $10 \mu \mathrm{M}$ ). See Figure 4A, C for graph of results. ns: not significant $(p>0.05){ }^{*} p<0.05,{ }^{\star *} p<0.01,{ }^{* \star} p<$ 0.001.

Table S5. Tukey's multiple comparison test results for Synapses/Neurite Length. Tables show the results of each pairwise comparison for hiPSC-neurons treated for one (left) or seven days (right) with DMSO alone, $25 \mu \mathrm{M}$ blebbistatin, single ARVs, or combinations of ARVs (each at $10 \mu \mathrm{M})$. See Figure 4B, D for graph of results. ns: not significant $(p>0.05){ }^{*} p<0.05,{ }^{* *} p<$ $0.01,{ }^{* * *} p<0.001$

Table S6. Tukey's multiple comparison test results for percent neurons with calcium transient activity. Tables show the results of each pairwise comparison for hiPSC-neurons treated for one (left) or seven days (right) with DMSO alone, single ARVs, or combinations of ARVs (each at $10 \mu \mathrm{M})$. See Figure 6A, D for graph of results. ns: not significant $(p>0.05){ }^{*} p<$ $0.05,{ }^{* *} p<0.01,{ }^{* * *} p<0.001$.

Table S7. Tukey's multiple comparison test results for calcium transient event frequency. Tables show the results of each pairwise comparison for hiPSC-neurons treated for one (left) or 
seven days (right) with DMSO alone, single ARVs, or combinations of ARVs (each at $10 \mu \mathrm{M})$. See

Figure $6 B, E$ for graph of results. ns: not significant $(p>0.05){ }^{*} p<0.05,{ }^{* *} p<0.01,{ }^{* * *} p<0.001$.

Table S8. Tukey's multiple comparison test results for calcium transient mean peak amplitude. Tables show the results of each pairwise comparison for hiPSC-neurons treated for one (left) or seven days (right) with DMSO alone, single ARVs, or combinations of ARVs (each at $10 \mu \mathrm{M})$. See Figure $6 \mathrm{C}, \mathrm{F}$ for graph of results. ns: not significant $(p>0.05){ }^{*} p<0.05,{ }^{\star *} p<0.01$, ${ }^{* * *} p<0.001$. 


\section{References}

1. Global HIV \& AIDS statistics - 2019 fact sheet. Vol. 2020 (UNAIDS).

2. $\quad$ FDA. Attacking AIDS with a 'Cocktail' Therapy: Drug Combo Sends Deaths Plummeting. in HIV/AIDS News (1999).

3. Antiretroviral Therapy Cohort, C. Survival of HIV-positive patients starting antiretroviral therapy between 1996 and 2013: a collaborative analysis of cohort studies. Lancet HIV 4, e349-e356 (2017).

4. Cohen, M.S., et al. Antiretroviral Therapy for the Prevention of HIV-1 Transmission. N Engl J Med 375, 830-839 (2016).

5. Rodger, A.J., et al. Sexual Activity Without Condoms and Risk of HIV Transmission in Serodifferent Couples When the HIV-Positive Partner Is Using Suppressive Antiretroviral Therapy. JAMA 316, 171-181 (2016).

6. Grant, R.M., et al. Preexposure chemoprophylaxis for HIV prevention in men who have sex with men. N Engl J Med 363, 2587-2599 (2010).

7. Baeten, J.M., et al. Antiretroviral prophylaxis for HIV prevention in heterosexual men and women. N Engl J Med 367, 399-410 (2012).

8. Choopanya, K., et al. Antiretroviral prophylaxis for HIV infection in injecting drug users in Bangkok, Thailand (the Bangkok Tenofovir Study): a randomised, double-blind, placebocontrolled phase 3 trial. Lancet 381, 2083-2090 (2013).

9. Hurst, S.A., Appelgren, K.E. \& Kourtis, A.P. Prevention of mother-to-child transmission of HIV type 1: the role of neonatal and infant prophylaxis. Expert Rev Anti Infect Ther 13, 169-181 (2015).

10. McCormack, S.A. \& Best, B.M. Protecting the fetus against HIV infection: a systematic review of placental transfer of antiretrovirals. Clin Pharmacokinet 53, 989-1004 (2014).

11. Sacktor, N. The epidemiology of human immunodeficiency virus-associated neurological disease in the era of highly active antiretroviral therapy. J Neuroviro/ 8 Suppl 2, 115-121 (2002).

12. Sacktor, N., et al. HIV-associated neurologic disease incidence changes:: Multicenter AIDS Cohort Study, 1990-1998. Neurology 56, 257-260 (2001).

13. d'Arminio Monforte, A., et al. Changing incidence of central nervous system diseases in the EuroSIDA cohort. Ann Neurol 55, 320-328 (2004).

14. Committee, U.K.C.H.C.S.S., et al. HIV-associated central nervous system diseases in the recent combination antiretroviral therapy era. Eur J Neurol 18, 527-534 (2011).

15. Heaton, R.K., et al. HIV-associated neurocognitive disorders persist in the era of potent antiretroviral therapy: CHARTER Study. Neurology 75, 2087-2096 (2010).

16. Cysique, L.A., Maruff, P. \& Brew, B.J. Prevalence and pattern of neuropsychological impairment in human immunodeficiency virus-infected/acquired immunodeficiency syndrome (HIV/AIDS) patients across pre- and post-highly active antiretroviral therapy eras: a combined study of two cohorts. J Neurovirol 10, 350-357 (2004).

17. Smail, R.C. \& Brew, B.J. HIV-associated neurocognitive disorder. Handb Clin Neurol 152, 75-97 (2018).

18. Saylor, D., et al. HIV-associated neurocognitive disorder - pathogenesis and prospects for treatment. Nat Rev Neurol 12, 309 (2016).

19. Heaton, R.K., et al. Neuropsychological impairment in human immunodeficiency virusinfection: implications for employment. HNRC Group. HIV Neurobehavioral Research Center. Psychosom Med 56, 8-17 (1994).

20. Brew, B.J. Benefit or toxicity from neurologically targeted antiretroviral therapy? Clin Infect Dis 50, 930-932 (2010). 
21. Gorman, A.A., Foley, J.M., Ettenhofer, M.L., Hinkin, C.H. \& van Gorp, W.G. Functional consequences of HIV-associated neuropsychological impairment. Neuropsychol Rev 19, 186-203 (2009).

22. Grant, I., et al. Asymptomatic HIV-associated neurocognitive impairment increases risk for symptomatic decline. Neurology 82, 2055-2062 (2014).

23. Heaton, R.K., et al. Neurocognitive change in the era of HIV combination antiretroviral therapy: the longitudinal CHARTER study. Clin Infect Dis 60, 473-480 (2015).

24. Underwood, J., Robertson, K.R. \& Winston, A. Could antiretroviral neurotoxicity play a role in the pathogenesis of cognitive impairment in treated HIV disease? AIDS 29, 253-261 (2015).

25. Akay, C., et al. Antiretroviral drugs induce oxidative stress and neuronal damage in the central nervous system. J Neurovirol 20, 39-53 (2014).

26. Robertson, K., Liner, J. \& Meeker, R.B. Antiretroviral neurotoxicity. J Neurovirol 18, 388399 (2012).

27. Zhang, Y., et al. Long-term exposure of mice to nucleoside analogues disrupts mitochondrial DNA maintenance in cortical neurons. PLoS One 9, e85637 (2014).

28. Stern, A.L., et al. Differential Effects of Antiretroviral Drugs on Neurons In Vitro: Roles for Oxidative Stress and Integrated Stress Response. J Neuroimmune Pharmacol 13, 64-76 (2018).

29. Schweinsburg, B.C., et al. Brain mitochondrial injury in human immunodeficiency virusseropositive $(\mathrm{HIV}+)$ individuals taking nucleoside reverse transcriptase inhibitors. $J$ Neurovirol 11, 356-364 (2005).

30. Jernigan, T.L., et al. Clinical factors related to brain structure in HIV: the CHARTER study. J Neurovirol 17, 248-257 (2011).

31. Jensen, B.K., et al. Altered Oligodendrocyte Maturation and Myelin Maintenance: The Role of Antiretrovirals in HIV-Associated Neurocognitive Disorders. J Neuropathol Exp Neurol 74, 1093-1118 (2015).

32. Chang, L., Yakupov, R., Nakama, H., Stokes, B. \& Ernst, T. Antiretroviral treatment is associated with increased attentional load-dependent brain activation in HIV patients. $J$ Neuroimmune Pharmacol 3, 95-104 (2008).

33. Tozzi, V., et al. Changes in cognition during antiretroviral therapy: comparison of 2 different ranking systems to measure antiretroviral drug efficacy on HIV-associated neurocognitive disorders. J Acquir Immune Defic Syndr 52, 56-63 (2009).

34. Santos, G.M.A., et al. Cross-Sectional and Cumulative Longitudinal Central Nervous System Penetration Effectiveness Scores Are Not Associated With Neurocognitive Impairment in a Well Treated Aging Human Immunodeficiency Virus-Positive Population in Switzerland. Open Forum Infect Dis 6, ofz277 (2019).

35. Marra, C.M., et al. Impact of combination antiretroviral therapy on cerebrospinal fluid HIV RNA and neurocognitive performance. AIDS 23, 1359-1366 (2009).

36. Kahouadji, Y., et al. Cognitive function after several years of antiretroviral therapy with stable central nervous system penetration score. HIV Med 14, 311-315 (2013).

37. Caniglia, E.C., et al. Antiretroviral penetration into the CNS and incidence of AIDS-defining neurologic conditions. Neurology 83, 134-141 (2014).

38. Robertson, K.R., et al. Neurocognitive effects of treatment interruption in stable HIVpositive patients in an observational cohort. Neurology 74, 1260-1266 (2010).

39. Dahabieh, M.S., Battivelli, E. \& Verdin, E. Understanding HIV latency: the road to an HIV cure. Annu Rev Med 66, 407-421 (2015).

40. Siliciano, R.F. \& Greene, W.C. HIV latency. Cold Spring Harb Perspect Med 1, a007096 (2011).

41. Cohen, S., et al. Poorer cognitive performance in perinatally HIV-infected children versus healthy socioeconomically matched controls. Clin Infect Dis 60, 1111-1119 (2015). 
42. Van den Hof, M., et al. Lower IQ and poorer cognitive profiles in treated perinatally HIVinfected children is irrespective of having a background of international adoption. PLoS One 14, e0224930 (2019).

43. Crowell, C.S., Malee, K.M., Yogev, R. \& Muller, W.J. Neurologic disease in HIV-infected children and the impact of combination antiretroviral therapy. Rev Med Virol 24, 316-331 (2014).

44. Sherr, L., Croome, N., Parra Castaneda, K. \& Bradshaw, K. A systematic review of psychological functioning of children exposed to HIV: using evidence to plan for tomorrow's HIV needs. AIDS Behav 18, 2059-2074 (2014).

45. Brown, L.A., et al. Efavirenz promotes beta-secretase expression and increased Abeta140,42 via oxidative stress and reduced microglial phagocytosis: implications for HIV associated neurocognitive disorders (HAND). PLoS One 9, e95500 (2014).

46. Gannon, P.J., et al. HIV Protease Inhibitors Alter Amyloid Precursor Protein Processing via beta-Site Amyloid Precursor Protein Cleaving Enzyme-1 Translational Up-Regulation. Am J Pathol 187, 91-109 (2017).

47. Giunta, B., et al. Antiretroviral medications disrupt microglial phagocytosis of beta-amyloid and increase its production by neurons: implications for HIV-associated neurocognitive disorders. Mol Brain 4, 23 (2011).

48. Fields, J.A., Swinton, M.K., Soontornniyomkij, B., Carson, A. \& Achim, C.L. Beta amyloid levels in cerebrospinal fluid of HIV-infected people vary by exposure to antiretroviral therapy. AIDS 34, 1001-1007 (2020).

49. Xu, J. \& Ikezu, T. The comorbidity of HIV-associated neurocognitive disorders and Alzheimer's disease: a foreseeable medical challenge in post-HAART era. $J$ Neuroimmune Pharmacol 4, 200-212 (2009).

50. McDonough, P.M., Prigozhina, N.L., Basa, R.C.B. \& Price, J.H. Assay of Calcium Transients and Synapses in Rat Hippocampal Neurons by Kinetic Image Cytometry and High-Content Analysis: An In Vitro Model System for Postchemotherapy Cognitive Impairment. Assay Drug Dev Technol 15, 220-236 (2017).

51. Hunsberger, J.G., et al. Induced Pluripotent Stem Cell Models to Enable In Vitro Models for Screening in the Central Nervous System. Stem Cells Dev 24, 1852-1864 (2015).

52. Miki, D., et al. Characterization of Functional Primary Cilia in Human Induced Pluripotent Stem Cell-Derived Neurons. Neurochem Res 44, 1736-1744 (2019).

53. Sherman, S.P. \& Bang, A.G. High-throughput screen for compounds that modulate neurite growth of human induced pluripotent stem cell-derived neurons. Dis Model Mech 11(2018).

54. Urban, N. \& Guillemot, F. Neurogenesis in the embryonic and adult brain: same regulators, different roles. Front Cell Neurosci 8, 396 (2014).

55. Hollands, C., Bartolotti, N. \& Lazarov, O. Alzheimer's Disease and Hippocampal Adult Neurogenesis; Exploring Shared Mechanisms. Front Neurosci 10, 178 (2016).

56. Farhy, C., et al. Improving drug discovery using image-based multiparametric analysis of the epigenetic landscape. Elife 8(2019).

57. Limouze, J., Straight, A.F., Mitchison, T. \& Sellers, J.R. Specificity of blebbistatin, an inhibitor of myosin II. J Muscle Res Cell Motil 25, 337-341 (2004).

58. Straight, A.F., et al. Dissecting temporal and spatial control of cytokinesis with a myosin II Inhibitor. Science 299, 1743-1747 (2003).

59. Davey, S., et al. Mother-to-Child HIV Transmission With In Utero Dolutegravir vs. Efavirenz in Botswana. J Acquir Immune Defic Syndr 84, 235-241 (2020).

60. Smetters, D., Majewska, A. \& Yuste, R. Detecting action potentials in neuronal populations with calcium imaging. Methods 18, 215-221 (1999).

61. Rosenberg, S.S. \& Spitzer, N.C. Calcium signaling in neuronal development. Cold Spring Harb Perspect Biol 3, a004259 (2011). 
62. Fink, C.C. \& Meyer, T. Molecular mechanisms of CaMKII activation in neuronal plasticity. Curr Opin Neurobiol 12, 293-299 (2002).

63. Wegierski, T. \& Kuznicki, J. Neuronal calcium signaling via store-operated channels in health and disease. Cell Calcium 74, 102-111 (2018).

64. Pfeiffer, E.R., Vega, R., McDonough, P.M., Price, J.H. \& Whittaker, R. Specific prediction of clinical QT prolongation by kinetic image cytometry in human stem cell derived cardiomyocytes. J Pharmacol Toxicol Methods 81, 263-273 (2016).

65. Hamilton, N.A., Pantelic, R.S., Hanson, K. \& Teasdale, R.D. Fast automated cell phenotype image classification. BMC Bioinformatics 8, 110 (2007).

66. Haralick, R.M., Shanmugam, K. \& Dinstein, I. Textural Features for Image Classification. IEEE Transactions on Systems, Man, and Cybernetics SMC-3, 610-621 (1973).

67. Gross, A.M., et al. Methylome-wide Analysis of Chronic HIV Infection Reveals Five-Year Increase in Biological Age and Epigenetic Targeting of HLA. Mol Cell 62, 157-168 (2016).

68. Nasi, M., et al. Ageing and inflammation in patients with HIV infection. Clin Exp Immunol 187, 44-52 (2017).

69. Shiels, M.S., Pfeiffer, R.M. \& Engels, E.A. Age at cancer diagnosis among persons with AIDS in the United States. Ann Intern Med 153, 452-460 (2010).

70. Autenrieth, C.S., et al. Global and regional trends of people living with HIV aged 50 and over: Estimates and projections for 2000-2020. PLoS One 13, e0207005 (2018).

71. Gong, Y., et al. Novel elvitegravir nanoformulation for drug delivery across the blood-brain barrier to achieve HIV-1 suppression in the CNS macrophages. Scientific reports 10, 3835 (2020).

72. Amano, M., et al. Novel Central Nervous System (CNS)-Targeting Protease Inhibitors for Drug-Resistant HIV Infection and HIV-Associated CNS Complications. Antimicrob Agents Chemother 63(2019).

73. Chaganti, J., et al. Imaging correlates of the blood-brain barrier disruption in HIVassociated neurocognitive disorder and therapeutic implications. AIDS 33, 1843-1852 (2019).

74. Ferrara, M., et al. Antiretroviral drug concentrations in brain tissue of adult decedents. AIDS 34, 1907-1914 (2020).

75. Allen, N.J. \& Lyons, D.A. Glia as architects of central nervous system formation and function. Science 362, 181-185 (2018).

76. Yang, Q.Q. \& Zhou, J.W. Neuroinflammation in the central nervous system: Symphony of glial cells. Glia 67, 1017-1035 (2019).

77. Nirzhor, S.S.R., Khan, R.I. \& Neelotpol, S. The Biology of Glial Cells and Their Complex Roles in Alzheimer's Disease: New Opportunities in Therapy. Biomolecules 8(2018).

78. Ryan, S.K., et al. Neuroinflammation and EIF2 Signaling Persist despite Antiretroviral Treatment in an hiPSC Tri-culture Model of HIV Infection. Stem Cell Reports 14, 991 (2020).

79. Apostolova, N., et al. Efavirenz and the CNS: what we already know and questions that need to be answered. J Antimicrob Chemother 70, 2693-2708 (2015).

80. Hakkers, C.S., et al. Objective and Subjective Improvement of Cognition After Discontinuing Efavirenz in Asymptomatic Patients: A Randomized Controlled Trial. J Acquir Immune Defic Syndr 80, e14-e22 (2019).

81. Lapadula, G., et al. Switching from efavirenz to rilpivirine improves sleep quality and selfperceived cognition but has no impact on neurocognitive performances. AIDS 34, 53-61 (2020).

82. Wallet, C., et al. Microglial Cells: The Main HIV-1 Reservoir in the Brain. Front Cell Infect Microbiol 9, 362 (2019).

83. Kaul, M. HIV's double strike at the brain: neuronal toxicity and compromised neurogenesis. Front Biosci 13, 2484-2494 (2008). 
84. Xu, P., et al. Combined Medication of Antiretroviral Drugs Tenofovir Disoproxil Fumarate, Emtricitabine, and Raltegravir Reduces Neural Progenitor Cell Proliferation In Vivo and In Vitro. J Neuroimmune Pharmacol 12, 682-692 (2017).

85. Demir, M. \& Laywell, E.D. Neurotoxic effects of AZT on developing and adult neurogenesis. Front Neurosci 9, 93 (2015).

86. Shiau, S., et al. Distinct epigenetic profiles in children with perinatally-acquired HIV on antiretroviral therapy. Sci Rep 9, 10495 (2019).

87. Letendre, S. Central nervous system complications in HIV disease: HIV-associated neurocognitive disorder. Top Antivir Med 19, 137-142 (2011).

88. Berger, J.R. \& Clifford, D.B. The relationship of CPE to HIV dementia: slain by an ugly fact? Neurology 83, 109-110 (2014).

89. Yuan, N.Y. \& Kaul, M. Beneficial and Adverse Effects of cART Affect Neurocognitive Function in HIV-1 Infection: Balancing Viral Suppression against Neuronal Stress and Injury. J Neuroimmune Pharmacol (2019). 
Table 1. Plasma concentrations of HIV antiretrovirals used in this study.

\begin{tabular}{cccc} 
Antiretroviral & Abbreviation & Drug class & Plasma Cmax \\
\hline Dolutegravir & DTG & INSTI & $8 \mu \mathrm{M}$ \\
Elvitegravir & EVG & INSTI & $3.5 \mu \mathrm{M}$ \\
Tenofovir disproxil fumarate & TDF & NRTI & $0.6 \mu \mathrm{M}$ \\
Emtricitabine & FTC & NRTI & $7 \mu \mathrm{M}$ \\
\hline
\end{tabular}

INSTI: integrase strand transfer inhibitor; NRTI: nucleoside/nucleotide reverse transcription inhibitor. Data taken from Food and Drug Administration New Drug Applications for each antiretroviral. 
bioRxiv preprint doi: https://doi.org/10.1101/2020.09.05.284422; this version posted January 11, 2021. The copyright holder for this preprint (which was not certified by peer review) is the author/funder, who has granted bioRxiv a license to display the preprint in perpetuity. It is made Figure 1 available under aCC-BY-NC-ND 4.0 International license.
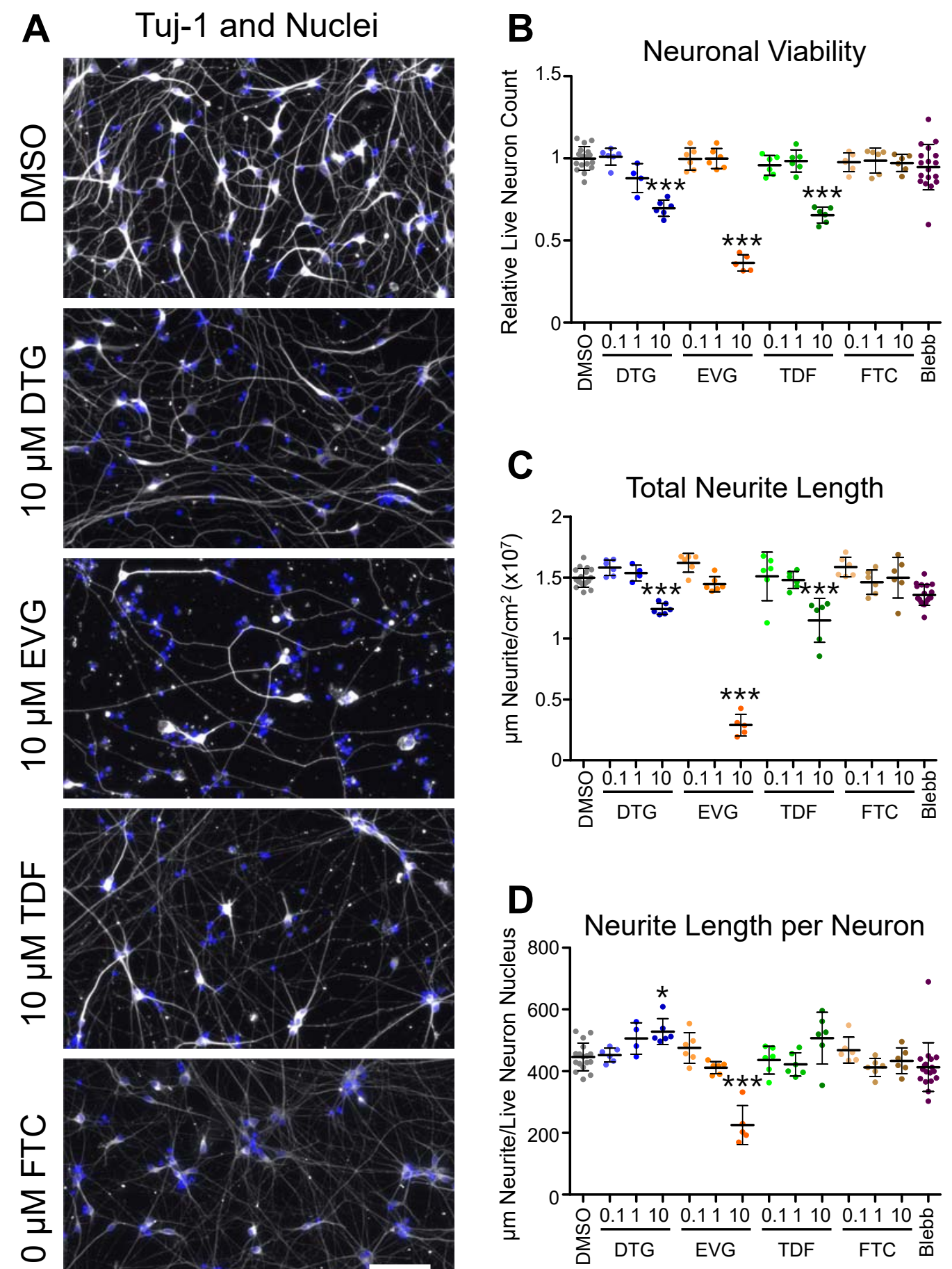
One Day Treatment A
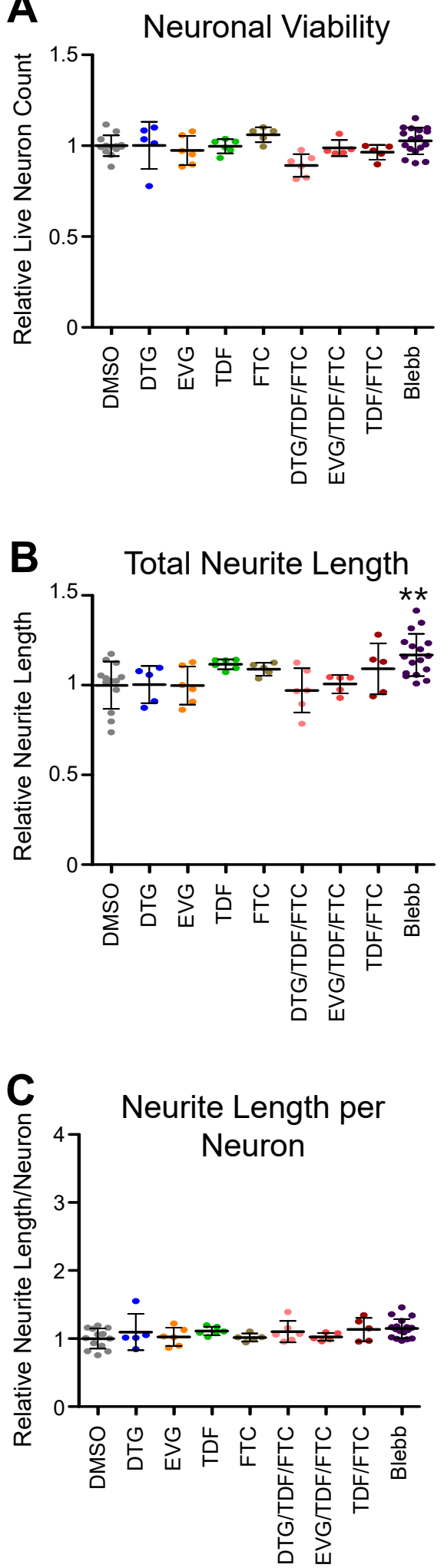

Seven Day Treatment
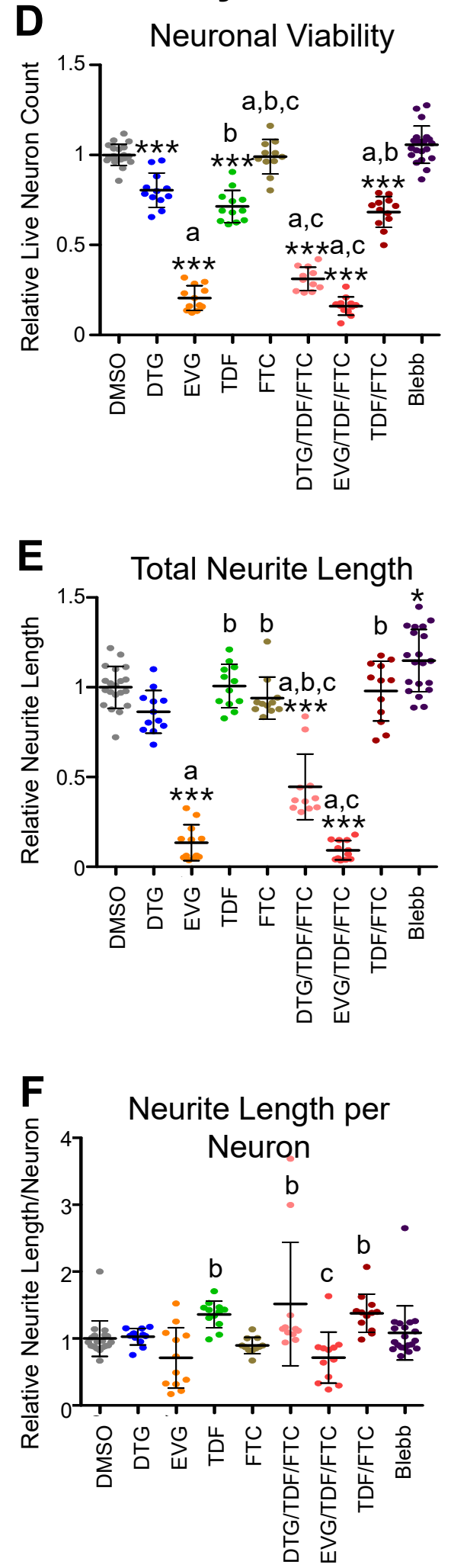


\section{Figure 3}
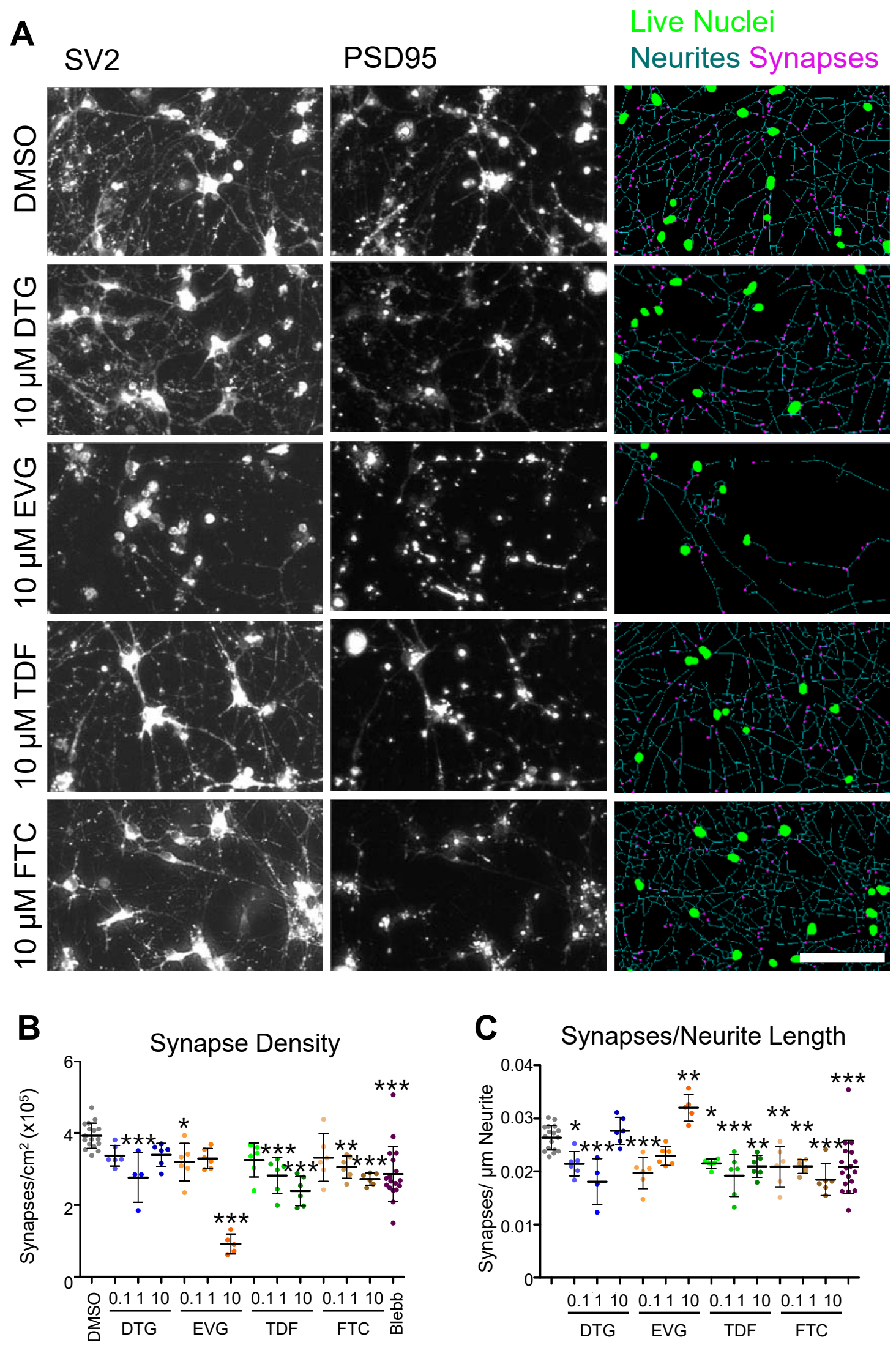

C Synapses/Neurite Length

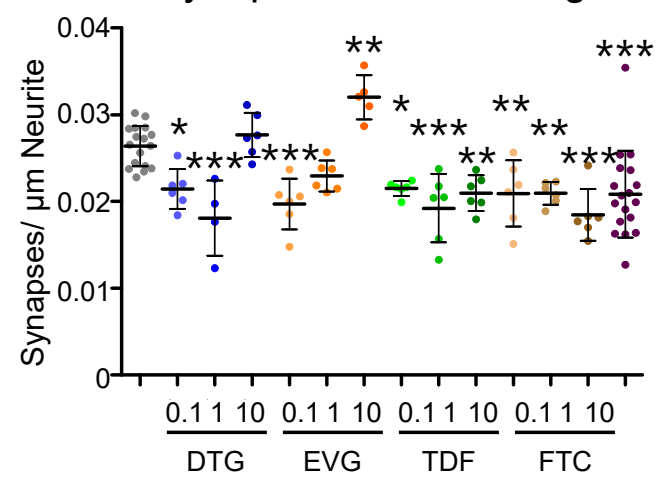




\section{Figure 4}
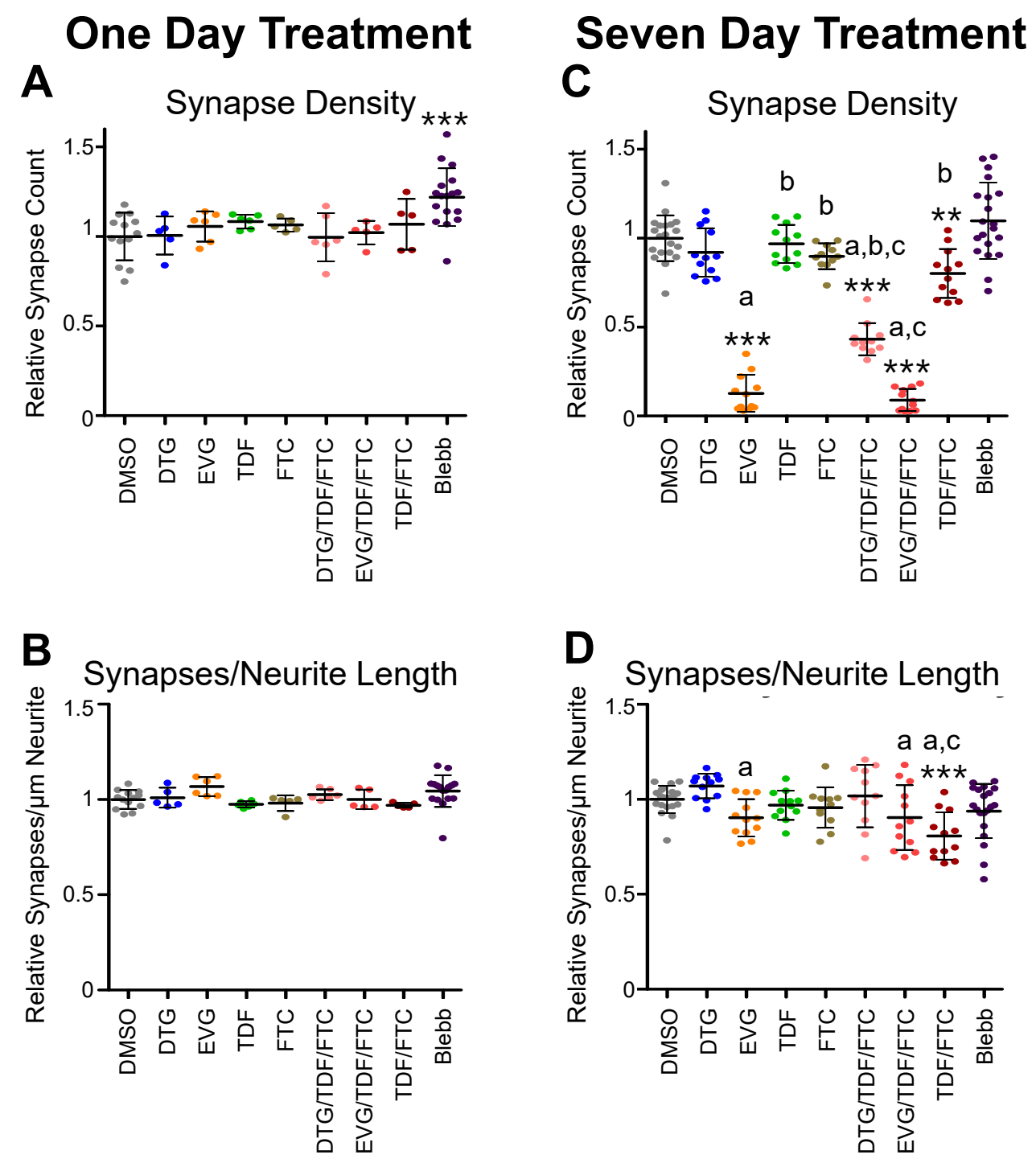
bioRxiv preprint doi: https://doi.org/10.1101/2020.09.05.284422; this version posted January 11,2021 . The copyright holder for this preprint (which was not certified by peer review) is the author/funder, who has granted bioRxiv a license to display the preprint in perpetuity. It is made available under aCC-BY-NC-ND 4.0 International license.

\section{Figure 5}
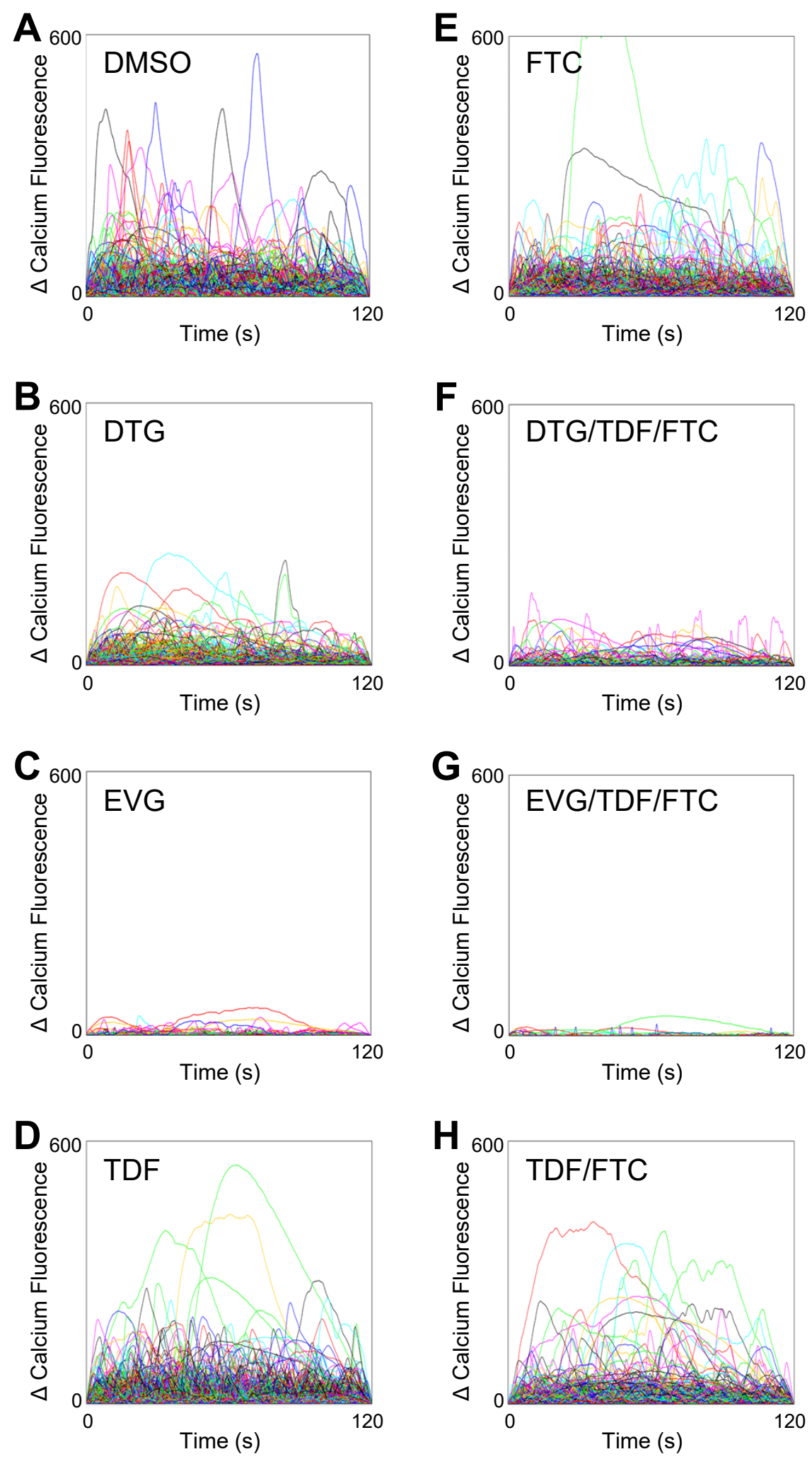
Figure 6

\section{One Day Treatment}

A

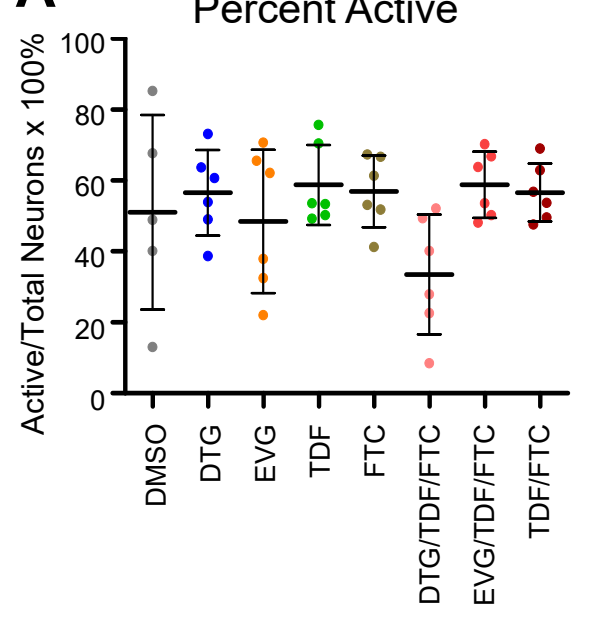

B
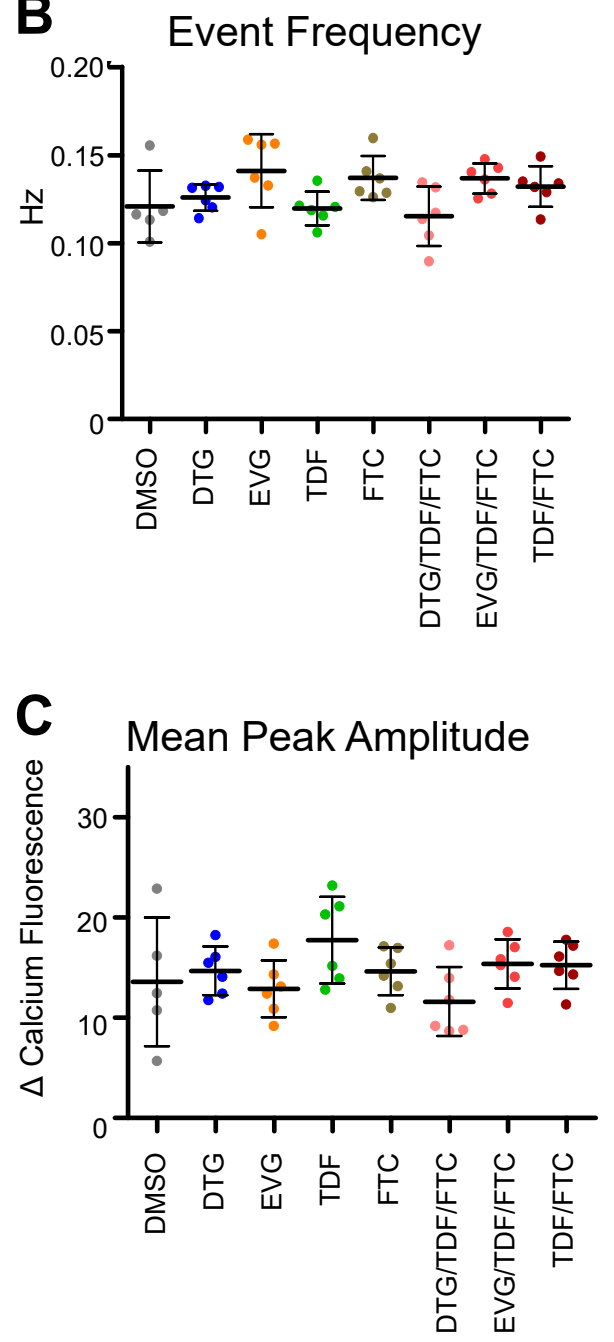

\section{Seven Day Treatment}

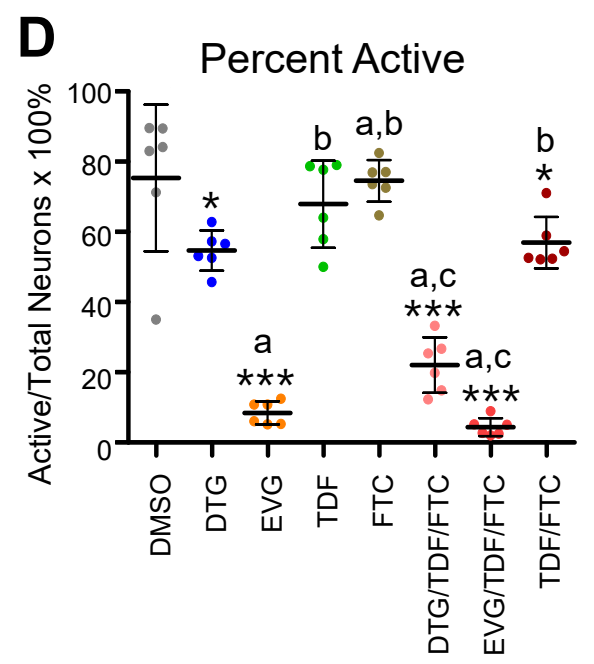

E Event Frequency
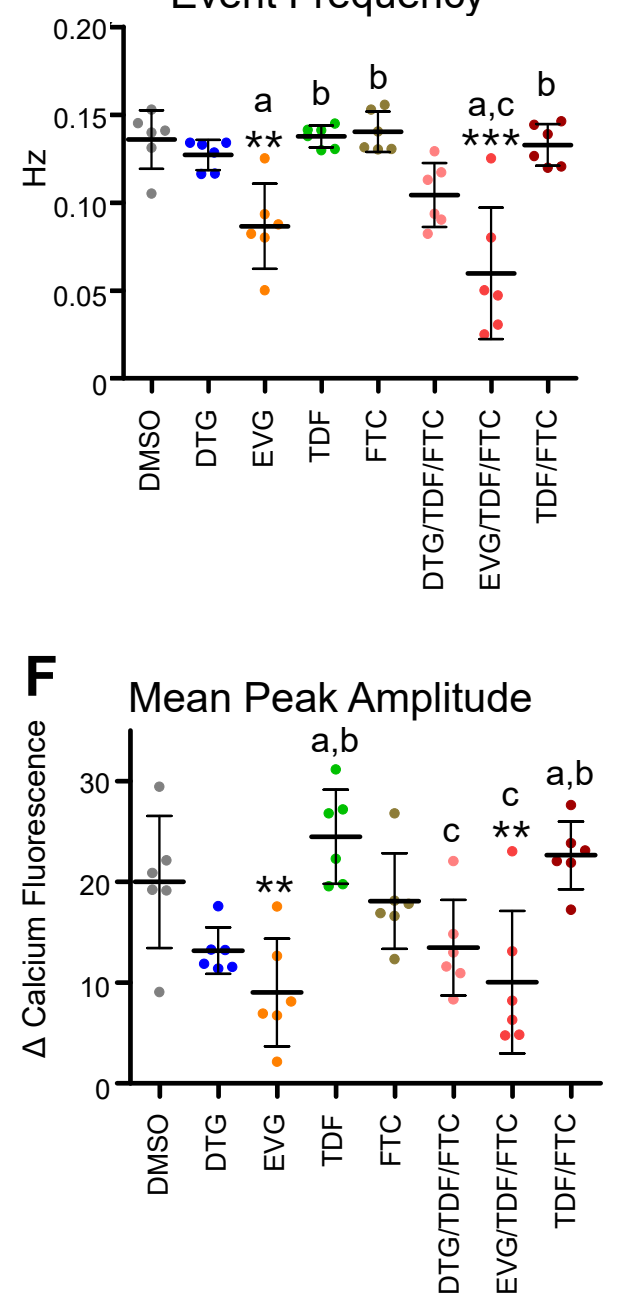


\section{Figure 7}
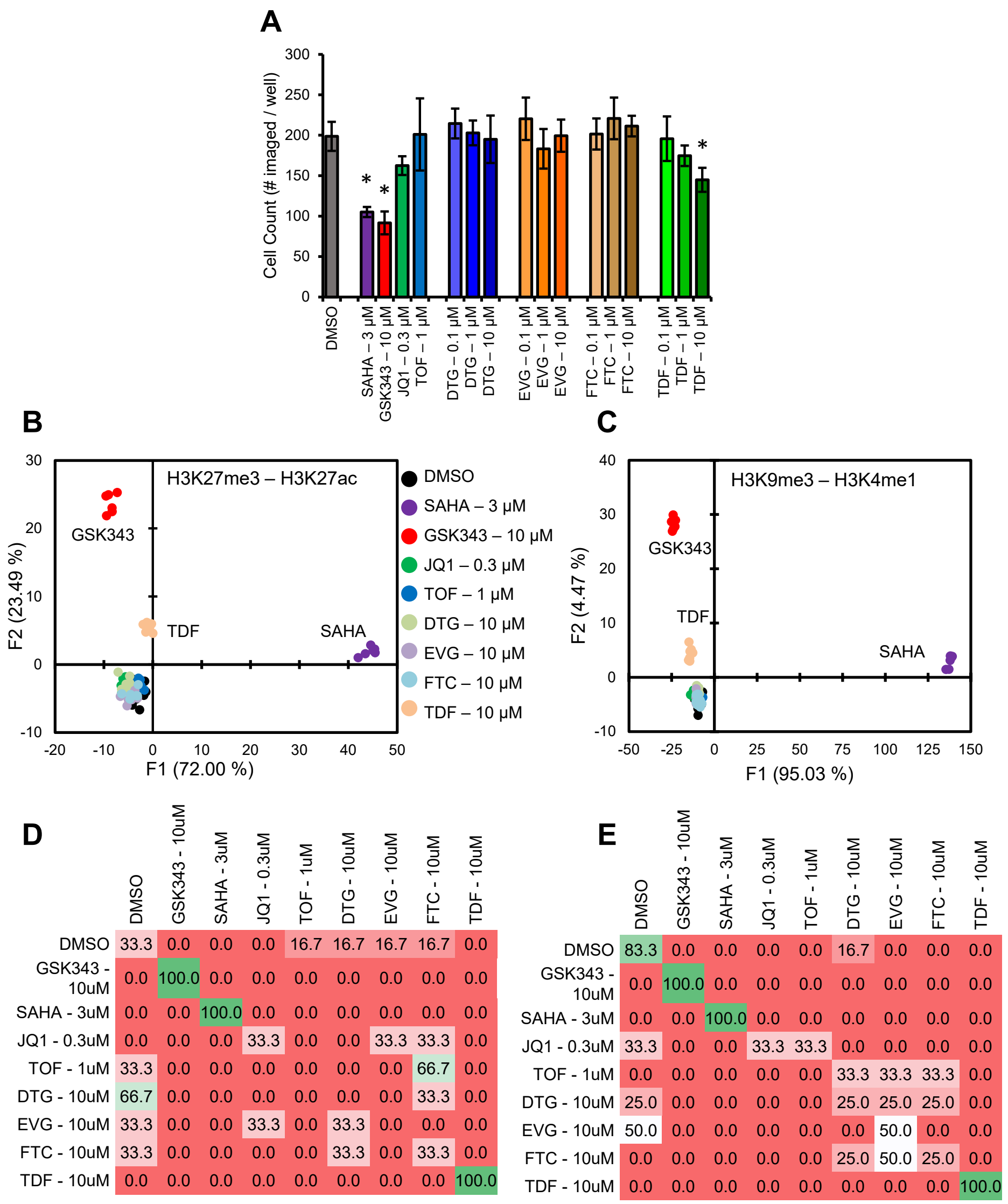


\section{Figure 8}

HIV antiretroviral effects on mature neurons

(1)Treatment

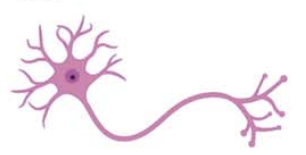

hiPSC-neurons

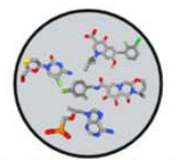

Antiretrovirals
(2) Assay

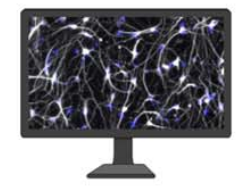

MNMNMNMN $\begin{aligned} & \text { Kinetic Image } \\ & \text { Cytometry@ }\end{aligned}$
(3) Results High content
analysis

Neurites +
synapses

Calcium
transients

HIV antiretroviral effects on human neural precusor cells

(1)Treatment

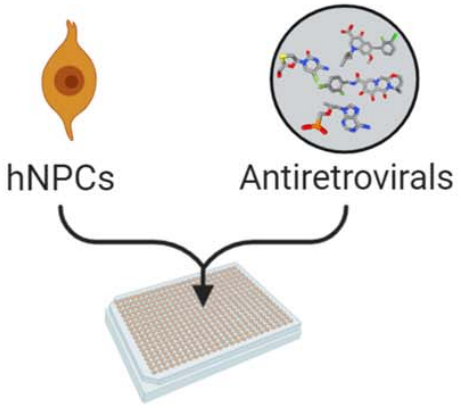

(2) Assay

(3) Results

MIEL epigenetics analysis

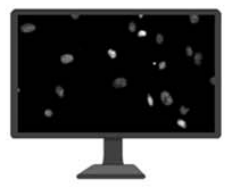

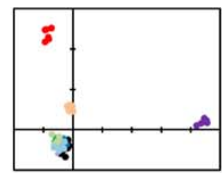

Viability

Altered histone modification pattern 


\section{Neuronal Viability}

\section{One Day Treatment}

\begin{tabular}{|c|c|c|}
\hline $\begin{array}{l}\text { Tukey's Multiple } \\
\text { Comparison Test }\end{array}$ & \begin{tabular}{|l} 
Mean \\
Difference
\end{tabular} & Significance \\
\hline DMSO vs DTG & -0.002360 & ns \\
\hline DMSO vs EVG & 0.02578 & $\mathrm{~ns}$ \\
\hline DMSO vs TDF & 0.002801 & ns \\
\hline DMSO vs FTC & -0.06047 & ns \\
\hline DMSO vs DTG TDF FTC & 0.1080 & $\mathrm{~ns}$ \\
\hline DMSO vs EVG TDF FTC & 0.01235 & ns \\
\hline DMSO vs TDF FTC & 0.03525 & $\mathrm{~ns}$ \\
\hline DMSO vs Blebb & -0.02633 & $\mathrm{~ns}$ \\
\hline DTG vs EVG & 0.02814 & $\mathrm{~ns}$ \\
\hline DTG vs TDF & 0.005161 & $\mathrm{~ns}$ \\
\hline DTG vs FTC & -0.05811 & $\mathrm{~ns}$ \\
\hline DTG vs DTG TDF FTC & 0.1104 & ns \\
\hline DTG vs EVG TDF FTC & 0.01471 & $\mathrm{~ns}$ \\
\hline DTG vs TDF FTC & 0.03761 & $\mathrm{~ns}$ \\
\hline DTG vs Blebb & -0.02397 & $\mathrm{~ns}$ \\
\hline EVG vs TDF & -0.02298 & ns \\
\hline EVG vs FTC & -0.08625 & ns \\
\hline EVG vs DTG TDF FTC & 0.08225 & $\mathrm{~ns}$ \\
\hline EVG vs EVG TDF FTC & -0.01343 & $\mathrm{~ns}$ \\
\hline EVG vs TDF FTC & 0.009474 & $\mathrm{~ns}$ \\
\hline EVG vs Blebb & -0.05211 & $\mathrm{~ns}$ \\
\hline TDF vs FTC & -0.06328 & ns \\
\hline TDF vs DTG TDF FTC & 0.1052 & $\mathrm{~ns}$ \\
\hline TDF vs EVG TDF FTC & 0.009544 & ns \\
\hline TDF vs TDF FTC & 0.03245 & $\mathrm{~ns}$ \\
\hline TDF vs Blebb & -0.02913 & ns \\
\hline FTC vs DTG TDF FTC & 0.1685 & ** \\
\hline FTC vs EVG TDF FTC & 0.07282 & $\mathrm{~ns}$ \\
\hline FTC vs TDF FTC & 0.09573 & $\mathrm{~ns}$ \\
\hline FTC vs Blebb & 0.03414 & $\mathrm{~ns}$ \\
\hline DTG TDF FTC vs EVG TDF FTC & -0.09568 & $\mathrm{~ns}$ \\
\hline DTG TDF FTC vs TDF FTC & -0.07277 & $\mathrm{~ns}$ \\
\hline DTG TDF FTC vs Blebb & -0.1344 & $* *$ \\
\hline EVG TDF FTC vs TDF FTC & 0.02291 & $\mathrm{~ns}$ \\
\hline EVG TDF FTC vs Blebb & -0.03868 & $\mathrm{~ns}$ \\
\hline TDF FTC vs Blebb & -0.06158 & $\mathrm{~ns}$ \\
\hline
\end{tabular}

\section{Seven Day Treatment}

\begin{tabular}{|c|c|c|}
\hline $\begin{array}{l}\text { Tukey's Multiple } \\
\text { Comparison Test }\end{array}$ & \begin{tabular}{|l} 
Mean \\
Difference
\end{tabular} & Significance \\
\hline DMSO vs DTG & 0.1952 & *** \\
\hline DMSO vs EVG & 0.7947 & *** \\
\hline DMSO vs TDF & 0.2858 & *** \\
\hline DMSO vs FTC & 0.009897 & $\mathrm{~ns}$ \\
\hline DMSO vs DTG TDF FTC & 0.6876 & 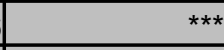 \\
\hline DMSO vs EVG TDF FTC & 0.8388 & *** \\
\hline DMSO vs TDF FTC & 0.3164 & *** \\
\hline DMSO vs Blebb & -0.05650 & $\mathrm{~ns}$ \\
\hline DTG vs EVG & 0.5994 & *** \\
\hline DTG vs TDF & 0.09056 & $\mathrm{~ns}$ \\
\hline DTG vs FTC & -0.1853 & $* \star \star$ \\
\hline DTG vs DTG TDF FTC & 0.4923 & $* * *$ \\
\hline DTG vs EVG TDF FTC & 0.6436 & *** \\
\hline DTG vs TDF FTC & 0.1212 & \\
\hline DTG vs Blebb & -0.2517 & *** \\
\hline EVG vs TDF & -0.5089 & 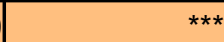 \\
\hline EVG vs FTC & -0.7848 & *** \\
\hline EVG vs DTG TDF FTC & -0.1071 & $\mathrm{~ns}$ \\
\hline EVG vs EVG TDF FTC & 0.04412 & $\mathrm{~ns}$ \\
\hline EVG vs TDF FTC & -0.4783 & 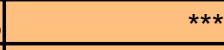 \\
\hline EVG vs Blebb & -0.8512 & 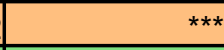 \\
\hline TDF vs FTC & -0.2759 & *** \\
\hline TDF vs DTG TDF FTC & 0.4018 & $* * *$ \\
\hline TDF vs EVG TDF FTC & 0.5530 & $* \star *$ \\
\hline TDF vs TDF FTC & 0.03061 & $\mathrm{~ns}$ \\
\hline TDF vs Blebb & -0.3423 & *** \\
\hline FTC vs DTG TDF FTC & 0.6777 & 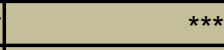 \\
\hline FTC vs EVG TDF FTC & 0.8289 & *** \\
\hline FTC vs TDF FTC & 0.3065 & *** \\
\hline FTC vs Blebb & -0.06640 & $\mathrm{~ns}$ \\
\hline DTG TDF FTC vs EVG TDF FTC & 0.1512 & *** \\
\hline DTG TDF FTC vs TDF FTC & -0.3712 & $* * *$ \\
\hline DTG TDF FTC vs Blebb & -0.7441 & *** \\
\hline EVG TDF FTC vs TDF FTC & -0.5224 & $* * *$ \\
\hline EVG TDF FTC vs Blebb & -0.8953 & $* * *$ \\
\hline TDF FTC vs Blebb & -0.3729 & 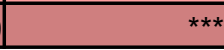 \\
\hline
\end{tabular}




\section{Total Neurite Length}

\section{One Day Treatment}

\begin{tabular}{|c|c|c|}
\hline $\begin{array}{l}\text { Tukey's Multiple } \\
\text { Comparison Test }\end{array}$ & \begin{tabular}{|l} 
Mean \\
Difference
\end{tabular} & Significance \\
\hline DMSO vs DTG & -0.003525 & ns \\
\hline DMSO vs EVG & 0.001910 & ns \\
\hline DMSO vs TDF & -0.1167 & $\mathrm{~ns}$ \\
\hline DMSO vs FTC & -0.08899 & $\mathrm{~ns}$ \\
\hline DMSO vs DTG TDF FTC & 0.02920 & $\mathrm{~ns}$ \\
\hline DMSO vs EVG TDF FTC & -0.006731 & ns \\
\hline DMSO vs TDF FTC & -0.09129 & $\mathrm{~ns}$ \\
\hline DMSO vs Blebb & -0.1680 & ** \\
\hline DTG vs EVG & 0.005434 & ns \\
\hline DTG vs TDF & -0.1132 & ns \\
\hline DTG vs FTC & -0.08547 & ns \\
\hline DTG vs DTG TDF FTC & 0.03272 & $\mathrm{~ns}$ \\
\hline DTG vs EVG TDF FTC & -0.003206 & $\mathrm{~ns}$ \\
\hline DTG vs TDF FTC & -0.08777 & $\mathrm{~ns}$ \\
\hline DTG vs Blebb & -0.1645 & $\mathrm{~ns}$ \\
\hline EVG vs TDF & -0.1186 & $\mathrm{~ns}$ \\
\hline EVG vs FTC & -0.09090 & $\mathrm{~ns}$ \\
\hline EVG vs DTG TDF FTC & 0.02729 & $\mathrm{~ns}$ \\
\hline EVG vs EVG TDF FTC & -0.008641 & ns \\
\hline EVG vs TDF FTC & -0.09320 & $\mathrm{~ns}$ \\
\hline EVG vs Blebb & -0.1699 & \\
\hline TDF vs FTC & 0.02772 & $\mathrm{~ns}$ \\
\hline TDF vs DTG TDF FTC & 0.1459 & $\mathrm{~ns}$ \\
\hline TDF vs EVG TDF FTC & 0.1100 & $\mathrm{~ns}$ \\
\hline TDF vs TDF FTC & 0.02542 & ns \\
\hline TDF vs Blebb & -0.05132 & $\mathrm{~ns}$ \\
\hline FTC vs DTG TDF FTC & 0.1182 & $\mathrm{~ns}$ \\
\hline FTC vs EVG TDF FTC & 0.08226 & $\mathrm{~ns}$ \\
\hline FTC vs TDF FTC & -0.002303 & $\mathrm{~ns}$ \\
\hline FTC vs Blebb & -0.07904 & $\mathrm{~ns}$ \\
\hline DTG TDF FTC vs EVG TDF FTC & -0.03593 & $\mathrm{~ns}$ \\
\hline DTG TDF FTC vs TDF FTC & -0.1205 & $\mathrm{~ns}$ \\
\hline DTG TDF FTC vs Blebb & -0.1972 & ** \\
\hline EVG TDF FTC vs TDF FTC & -0.08456 & $\mathrm{~ns}$ \\
\hline EVG TDF FTC vs Blebb & -0.1613 & $\mathrm{~ns}$ \\
\hline TDF FTC vs Blebb & -0.07674 & ns \\
\hline
\end{tabular}

\section{Seven Day Treatment}

\begin{tabular}{|c|c|c|}
\hline \begin{tabular}{|l} 
Tukey's Multiple \\
Comparison Test
\end{tabular} & \begin{tabular}{|l} 
Mean \\
Difference
\end{tabular} & Significance \\
\hline DMSO vs DTG & 0.1369 & ns \\
\hline DMSO vs EVG & 0.8657 & *** \\
\hline DMSO vs TDF & -0.006719 & ns \\
\hline DMSO vs FTC & 0.06042 & ns \\
\hline DMSO vs DTG TDF FTC & 0.5543 & $* * \star$ \\
\hline DMSO vs EVG TDF FTC & 0.9078 & $* * \star$ \\
\hline DMSO vs TDF FTC & 0.02082 & ns \\
\hline DMSO vs Blebb & -0.1483 & \\
\hline DTG vs EVG & 0.7288 & $* * \star$ \\
\hline DTG vs TDF & -0.1436 & $\mathrm{~ns}$ \\
\hline DTG vs FTC & -0.07650 & $\mathrm{~ns}$ \\
\hline DTG vs DTG TDF FTC & 0.4174 & *** \\
\hline DTG vs EVG TDF FTC & 0.7708 & $* * \star$ \\
\hline DTG vs TDF FTC & -0.1161 & ns \\
\hline DTG vs Blebb & -0.2853 & $* * \star$ \\
\hline EVG vs TDF & -0.8724 & $* * \star$ \\
\hline EVG vs FTC & -0.8053 & 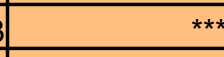 \\
\hline EVG vs DTG TDF FTC & -0.3114 & *** \\
\hline EVG vs EVG TDF FTC & 0.04204 & $\mathrm{~ns}$ \\
\hline EVG vs TDF FTC & -0.8449 & $* \star \star$ \\
\hline EVG vs Blebb & -1.014 & 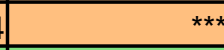 \\
\hline TDF vs FTC & 0.06714 & ns \\
\hline TDF vs DTG TDF FTC & 0.5610 & *** \\
\hline TDF vs EVG TDF FTC & 0.9145 & 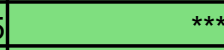 \\
\hline TDF vs TDF FTC & 0.02753 & $\mathrm{~ns}$ \\
\hline TDF vs Blebb & -0.1416 & ns \\
\hline FTC vs DTG TDF FTC & 0.4939 & *** \\
\hline FTC vs EVG TDF FTC & 0.8473 & *** \\
\hline FTC vs TDF FTC & -0.03961 & ns \\
\hline FTC vs Blebb & -0.2088 & ** \\
\hline DTG TDF FTC vs EVG TDF FTC & 0.3535 & *** \\
\hline DTG TDF FTC vs TDF FTC & -0.5335 & $* * \star$ \\
\hline DTG TDF FTC vs Blebb & -0.7026 & *** \\
\hline EVG TDF FTC vs TDF FTC & -0.8870 & $* * \star$ \\
\hline EVG TDF FTC vs Blebb & -1.056 & $* * \star$ \\
\hline TDF FTC vs Blebb & -0.1691 & \\
\hline
\end{tabular}




\section{Neurite Length Per Neuron}

\section{One Day Treatment}

\begin{tabular}{|c|c|c|}
\hline $\begin{array}{l}\text { Tukey's Multiple } \\
\text { Comparison Test }\end{array}$ & \begin{tabular}{|l|} 
Mean \\
Difference
\end{tabular} & Significance \\
\hline DMSO vs DTG & \begin{tabular}{|r|}
-0.09484 \\
\end{tabular} & ns \\
\hline DMSO vs EVG & -0.02655 & $\mathrm{~ns}$ \\
\hline DMSO vs TDF & -0.1107 & $\mathrm{~ns}$ \\
\hline DMSO vs FTC & -0.01540 & $\mathrm{~ns}$ \\
\hline DMSO vs DTG TDF FTC & -0.1021 & $\mathrm{~ns}$ \\
\hline DMSO vs EVG TDF FTC & -0.02473 & $\mathrm{~ns}$ \\
\hline DMSO vs TDF FTC & -0.1341 & $\mathrm{~ns}$ \\
\hline DMSO vs Blebb & -0.1484 & $\mathrm{~ns}$ \\
\hline DTG vs EVG & 0.06828 & $\mathrm{~ns}$ \\
\hline DTG vs TDF & -0.01582 & $\mathrm{~ns}$ \\
\hline DTG vs FTC & 0.07943 & $\mathrm{~ns}$ \\
\hline DTG vs DTG TDF FTC & -0.007285 & $\mathrm{~ns}$ \\
\hline DTG vs EVG TDF FTC & 0.07010 & $\mathrm{~ns}$ \\
\hline DTG vs TDF FTC & -0.03929 & $\mathrm{~ns}$ \\
\hline DTG vs Blebb & -0.05354 & $\mathrm{~ns}$ \\
\hline EVG vs TDF & -0.08410 & $\mathrm{~ns}$ \\
\hline EVG vs FTC & 0.01115 & $\mathrm{~ns}$ \\
\hline EVG vs DTG TDF FTC & -0.07557 & $\mathrm{~ns}$ \\
\hline EVG vs EVG TDF FTC & 0.001821 & $\mathrm{~ns}$ \\
\hline EVG vs TDF FTC & -0.1076 & ns \\
\hline EVG vs Blebb & -0.1218 & $\mathrm{~ns}$ \\
\hline TDF vs FTC & 0.09526 & $\mathrm{~ns}$ \\
\hline TDF vs DTG TDF FTC & 0.008536 & $\mathrm{~ns}$ \\
\hline TDF vs EVG TDF FTC & 0.08592 & $\mathrm{~ns}$ \\
\hline TDF vs TDF FTC & -0.02347 & $\mathrm{~ns}$ \\
\hline TDF vs Blebb & -0.03772 & $\mathrm{~ns}$ \\
\hline FTC vs DTG TDF FTC & -0.08672 & $\mathrm{~ns}$ \\
\hline FTC vs EVG TDF FTC & -0.009331 & $\mathrm{~ns}$ \\
\hline FTC vs TDF FTC & -0.1187 & $\mathrm{~ns}$ \\
\hline FTC vs Blebb & -0.1330 & $\mathrm{~ns}$ \\
\hline DTG TDF FTC vs EVG TDF FTC & 0.07739 & $\mathrm{~ns}$ \\
\hline DTG TDF FTC vs TDF FTC & -0.03200 & $\mathrm{~ns}$ \\
\hline DTG TDF FTC vs Blebb & -0.04625 & $\mathrm{~ns}$ \\
\hline EVG TDF FTC vs TDF FTC & -0.1094 & $\mathrm{~ns}$ \\
\hline EVG TDF FTC vs Blebb & -0.1236 & $\mathrm{~ns}$ \\
\hline TDF FTC vs Blebb & -0.01425 & $\mathrm{~ns}$ \\
\hline
\end{tabular}

\section{Seven Day Treatment}

\begin{tabular}{|c|c|c|}
\hline $\begin{array}{l}\text { Tukey's Multiple } \\
\text { Comparison Test }\end{array}$ & \begin{tabular}{|l|} 
Mean \\
Difference \\
\end{tabular} & Significance \\
\hline DMSO vs DTG & $\begin{array}{r}-0.02907 \\
\end{array}$ & ns \\
\hline DMSO vs EVG & 0.2885 & ns \\
\hline DMSO vs TDF & -0.3622 & ns \\
\hline DMSO vs FTC & 0.1027 & ns \\
\hline DMSO vs DTG TDF FTC & -0.5172 & \\
\hline DMSO vs EVG TDF FTC & 0.2840 & ns \\
\hline DMSO vs TDF FTC & -0.3790 & ns \\
\hline DMSO vs Blebb & -0.08370 & ns \\
\hline DTG vs EVG & 0.3175 & ns \\
\hline DTG vs TDF & -0.3331 & ns \\
\hline DTG vs FTC & 0.1317 & ns \\
\hline DTG vs DTG TDF FTC & -0.4881 & ns \\
\hline DTG vs EVG TDF FTC & 0.3131 & ns \\
\hline DTG vs TDF FTC & -0.3499 & ns \\
\hline DTG vs Blebb & -0.05463 & ns \\
\hline EVG vs TDF & -0.6507 & ** \\
\hline EVG vs FTC & -0.1858 & ns \\
\hline EVG vs DTG TDF FTC & -0.8056 & $* * \star$ \\
\hline EVG vs EVG TDF FTC & -0.004459 & $\mathrm{~ns}$ \\
\hline EVG vs TDF FTC & -0.6675 & ** \\
\hline EVG vs Blebb & -0.3722 & ns \\
\hline TDF vs FTC & 0.4649 & ns \\
\hline TDF vs DTG TDF FTC & -0.1550 & ns \\
\hline TDF vs EVG TDF FTC & 0.6462 & *夫 \\
\hline TDF vs TDF FTC & -0.01681 & ns \\
\hline TDF vs Blebb & 0.2785 & ns \\
\hline FTC vs DTG TDF FTC & -0.6198 & \\
\hline FTC vs EVG TDF FTC & 0.1813 & ns \\
\hline FTC vs TDF FTC & -0.4817 & ns \\
\hline FTC vs Blebb & -0.1864 & ns \\
\hline DTG TDF FTC vs EVG TDF FTC & 0.8012 & $* * *$ \\
\hline DTG TDF FTC vs TDF FTC & 0.1382 & ns \\
\hline DTG TDF FTC vs Blebb & 0.4335 & ns \\
\hline EVG TDF FTC vs TDF FTC & -0.6630 & ** \\
\hline EVG TDF FTC vs Blebb & -0.3677 & $\mathrm{~ns}$ \\
\hline TDF FTC vs Blebb & 0.2953 & ns \\
\hline
\end{tabular}


bioRxiv preprint doi: https://doi.org/10.1101/2020.09.05.284422; this version posted January 11, 2021. The copyright holder for this preprint

(which was not certified by peer review) is the author/funder, who has granted bioRxiv a license to display the preprint in perpetuity. It is made

Table S4 available under aCC-BY-NC-ND 4.0 International license.

\section{Synapse Density}

\section{One Day Treatment}

\begin{tabular}{|c|c|c|}
\hline \begin{tabular}{|l} 
Tukey's Multiple \\
Comparison Test
\end{tabular} & $\begin{array}{l}\text { Mean } \\
\text { Difference }\end{array}$ & Significance \\
\hline DMSO vs DTG & -0.005561 & ns \\
\hline DMSO vs EVG & -0.05608 & $\mathrm{~ns}$ \\
\hline DMSO vs TDF & -0.08320 & $\mathrm{~ns}$ \\
\hline DMSO vs FTC & -0.06424 & $\mathrm{~ns}$ \\
\hline DMSO vs DTG TDF FTC & 0.004011 & $\mathrm{~ns}$ \\
\hline DMSO vs EVG TDF FTC & -0.02199 & $\mathrm{~ns}$ \\
\hline DMSO vs TDF FTC & -0.06814 & $\mathrm{~ns}$ \\
\hline DMSO vs Blebb & -0.2197 & 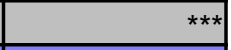 \\
\hline DTG vs EVG & -0.05051 & $\mathrm{~ns}$ \\
\hline DTG vs TDF & -0.07764 & $\mathrm{~ns}$ \\
\hline DTG vs FTC & -0.05868 & $\mathrm{~ns}$ \\
\hline DTG vs DTG TDF FTC & 0.009572 & $\mathrm{~ns}$ \\
\hline DTG vs EVG TDF FTC & -0.01643 & $\mathrm{~ns}$ \\
\hline DTG vs TDF FTC & -0.06258 & $\mathrm{~ns}$ \\
\hline DTG vs Blebb & -0.2142 & \\
\hline EVG vs TDF & -0.02713 & $\mathrm{~ns}$ \\
\hline EVG vs FTC & -0.008162 & $\mathrm{~ns}$ \\
\hline EVG vs DTG TDF FTC & 0.06009 & $\mathrm{~ns}$ \\
\hline EVG vs EVG TDF FTC & 0.03408 & $\mathrm{~ns}$ \\
\hline EVG vs TDF FTC & -0.01207 & $\mathrm{~ns}$ \\
\hline EVG vs Blebb & -0.1636 & $\mathrm{~ns}$ \\
\hline TDF vs FTC & 0.01897 & $\mathrm{~ns}$ \\
\hline TDF vs DTG TDF FTC & 0.08722 & $\mathrm{~ns}$ \\
\hline TDF vs EVG TDF FTC & 0.06121 & $\mathrm{~ns}$ \\
\hline TDF vs TDF FTC & 0.01506 & $\mathrm{~ns}$ \\
\hline TDF vs Blebb & -0.1365 & $\mathrm{~ns}$ \\
\hline FTC vs DTG TDF FTC & 0.06825 & $\mathrm{~ns}$ \\
\hline FTC vs EVG TDF FTC & 0.04225 & $\mathrm{~ns}$ \\
\hline FTC vs TDF FTC & -0.003904 & $\mathrm{~ns}$ \\
\hline FTC vs Blebb & -0.1555 & $\mathrm{~ns}$ \\
\hline DTG TDF FTC vs EVG TDF FTC & -0.02600 & $\mathrm{~ns}$ \\
\hline DTG TDF FTC vs TDF FTC & -0.07215 & $\mathrm{~ns}$ \\
\hline DTG TDF FTC vs Blebb & -0.2237 & $* *$ \\
\hline EVG TDF FTC vs TDF FTC & -0.04615 & $\mathrm{~ns}$ \\
\hline EVG TDF FTC vs Blebb & -0.1977 & $\mathrm{~ns}$ \\
\hline TDF FTC vs Blebb & -0.1516 & $\mathrm{~ns}$ \\
\hline
\end{tabular}

\section{Seven Day Treatment}

\begin{tabular}{|c|c|c|}
\hline $\begin{array}{l}\text { Tukey's Multiple } \\
\text { Comparison Test }\end{array}$ & \begin{tabular}{|l|} 
Mean \\
Difference
\end{tabular} & Significance \\
\hline DMSO vs DTG & 0.07950 & ns \\
\hline DMSO vs EVG & 0.8726 & *** \\
\hline DMSO vs TDF & 0.03189 & $\mathrm{~ns}$ \\
\hline DMSO vs FTC & 0.1014 & $\mathrm{~ns}$ \\
\hline DMSO vs DTG TDF FTC & 0.5669 & $\star * \star *$ \\
\hline DMSO vs EVG TDF FTC & 0.9095 & *** \\
\hline DMSO vs TDF FTC & 0.1984 & $* *$ \\
\hline DMSO vs Blebb & -0.09814 & $\mathrm{~ns}$ \\
\hline DTG vs EVG & 0.7931 & *** \\
\hline DTG vs TDF & -0.04760 & $\mathrm{~ns}$ \\
\hline DTG vs FTC & 0.02194 & $\mathrm{~ns}$ \\
\hline DTG vs DTG TDF FTC & 0.4874 & 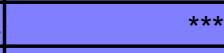 \\
\hline DTG vs EVG TDF FTC & 0.8300 & *** \\
\hline DTG vs TDF FTC & 0.1189 & $\mathrm{~ns}$ \\
\hline DTG vs Blebb & -0.1776 & \\
\hline EVG vs TDF & -0.8407 & *** \\
\hline EVG vs FTC & -0.7711 & $* * *$ \\
\hline EVG vs DTG TDF FTC & -0.3056 & *** \\
\hline EVG vs EVG TDF FTC & 0.03694 & ns \\
\hline EVG vs TDF FTC & -0.6742 & $* * \star$ \\
\hline EVG vs Blebb & -0.9707 & *** \\
\hline TDF vs FTC & 0.06954 & ns \\
\hline TDF vs DTG TDF FTC & 0.5350 & 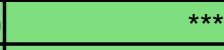 \\
\hline TDF vs EVG TDF FTC & 0.8776 & *** \\
\hline TDF vs TDF FTC & 0.1665 & $\mathrm{~ns}$ \\
\hline TDF vs Blebb & -0.1300 & ns \\
\hline FTC vs DTG TDF FTC & 0.4655 & *** \\
\hline FTC vs EVG TDF FTC & 0.8081 & *** \\
\hline FTC vs TDF FTC & 0.09693 & ns \\
\hline FTC vs Blebb & -0.1996 & ** \\
\hline DTG TDF FTC vs EVG TDF FTC & 0.3426 & *** \\
\hline DTG TDF FTC vs TDF FTC & -0.3685 & $* * *$ \\
\hline DTG TDF FTC vs Blebb & -0.6651 & *** \\
\hline EVG TDF FTC vs TDF FTC & -0.7111 & *** \\
\hline EVG TDF FTC vs Blebb & -1.008 & *** \\
\hline TDF FTC vs Blebb & -0.2965 & *** \\
\hline
\end{tabular}




\section{Synapses/Neurite Length}

\section{One Day Treatment}

\begin{tabular}{|c|c|c|}
\hline \begin{tabular}{|l} 
Tukey's Multiple \\
Comparison Test
\end{tabular} & $\begin{array}{l}\text { Mean } \\
\text { Difference }\end{array}$ & Significance \\
\hline DMSO vs DTG & -0.009334 & ns \\
\hline DMSO vs EVG & -0.06784 & $\mathrm{~ns}$ \\
\hline DMSO vs TDF & 0.02567 & $\mathrm{~ns}$ \\
\hline DMSO vs FTC & 0.01931 & $\mathrm{~ns}$ \\
\hline DMSO vs DTG TDF FTC & -0.02523 & $\mathrm{~ns}$ \\
\hline DMSO vs EVG TDF FTC & -0.0004753 & $\mathrm{~ns}$ \\
\hline DMSO vs TDF FTC & 0.03048 & $\mathrm{~ns}$ \\
\hline DMSO vs Blebb & -0.04413 & $\mathrm{~ns}$ \\
\hline DTG vs EVG & -0.05851 & $\mathrm{~ns}$ \\
\hline DTG vs TDF & 0.03500 & $\mathrm{~ns}$ \\
\hline DTG vs FTC & 0.02864 & $\mathrm{~ns}$ \\
\hline DTG vs DTG TDF FTC & -0.01589 & $\mathrm{~ns}$ \\
\hline DTG vs EVG TDF FTC & 0.008858 & $\mathrm{~ns}$ \\
\hline DTG vs TDF FTC & 0.03981 & $\mathrm{~ns}$ \\
\hline DTG vs Blebb & -0.03480 & $\mathrm{~ns}$ \\
\hline EVG vs TDF & 0.09351 & $\mathrm{~ns}$ \\
\hline EVG vs FTC & 0.08715 & $\mathrm{~ns}$ \\
\hline EVG vs DTG TDF FTC & 0.04262 & $\mathrm{~ns}$ \\
\hline EVG vs EVG TDF FTC & 0.06737 & $\mathrm{~ns}$ \\
\hline EVG vs TDF FTC & 0.09832 & $\mathrm{~ns}$ \\
\hline EVG vs Blebb & 0.02371 & $\mathrm{~ns}$ \\
\hline TDF vs FTC & -0.006361 & $\mathrm{~ns}$ \\
\hline TDF vs DTG TDF FTC & -0.05090 & $\mathrm{~ns}$ \\
\hline TDF vs EVG TDF FTC & -0.02615 & $\mathrm{~ns}$ \\
\hline TDF vs TDF FTC & 0.004810 & $\mathrm{~ns}$ \\
\hline TDF vs Blebb & -0.06980 & $\mathrm{~ns}$ \\
\hline FTC vs DTG TDF FTC & -0.04454 & $\mathrm{~ns}$ \\
\hline FTC vs EVG TDF FTC & -0.01979 & ns \\
\hline FTC vs TDF FTC & 0.01117 & $\mathrm{~ns}$ \\
\hline FTC vs Blebb & -0.06344 & $\mathrm{~ns}$ \\
\hline DTG TDF FTC vs EVG TDF FTC & 0.02475 & $\mathrm{~ns}$ \\
\hline DTG TDF FTC vs TDF FTC & 0.05571 & $\mathrm{~ns}$ \\
\hline DTG TDF FTC vs Blebb & -0.01891 & $\mathrm{~ns}$ \\
\hline EVG TDF FTC vs TDF FTC & 0.03096 & $\mathrm{~ns}$ \\
\hline EVG TDF FTC vs Blebb & -0.04366 & $\mathrm{~ns}$ \\
\hline TDF FTC vs Blebb & -0.07461 & $\mathrm{~ns}$ \\
\hline
\end{tabular}

\section{Seven Day Treatment}

\begin{tabular}{|c|c|c|}
\hline $\begin{array}{l}\text { Tukey's Multiple } \\
\text { Comparison Test }\end{array}$ & \begin{tabular}{|l} 
Mean \\
Difference
\end{tabular} & Significance \\
\hline DMSO vs DTG & -0.07061 & ns \\
\hline DMSO vs EVG & 0.09677 & $\mathrm{~ns}$ \\
\hline DMSO vs TDF & 0.03095 & $\mathrm{~ns}$ \\
\hline DMSO vs FTC & 0.04279 & $\mathrm{~ns}$ \\
\hline DMSO vs DTG TDF FTC & -0.01777 & $\mathrm{~ns}$ \\
\hline DMSO vs EVG TDF FTC & 0.09549 & ns \\
\hline DMSO vs TDF FTC & 0.1925 & *** \\
\hline DMSO vs Blebb & 0.06131 & $\mathrm{~ns}$ \\
\hline DTG vs EVG & 0.1674 & \\
\hline DTG vs TDF & 0.1016 & ns \\
\hline DTG vs FTC & 0.1134 & $\mathrm{~ns}$ \\
\hline DTG vs DTG TDF FTC & 0.05284 & ns \\
\hline DTG vs EVG TDF FTC & 0.1661 & \\
\hline DTG vs TDF FTC & 0.2632 & *** \\
\hline DTG vs Blebb & 0.1319 & $\mathrm{~ns}$ \\
\hline EVG vs TDF & -0.06582 & ns \\
\hline EVG vs FTC & -0.05398 & $\mathrm{~ns}$ \\
\hline EVG vs DTG TDF FTC & -0.1145 & $\mathrm{~ns}$ \\
\hline EVG vs EVG TDF FTC & -0.001282 & $\mathrm{~ns}$ \\
\hline EVG vs TDF FTC & 0.09577 & $\mathrm{~ns}$ \\
\hline EVG vs Blebb & -0.03546 & ns \\
\hline TDF vs FTC & 0.01184 & ns \\
\hline TDF vs DTG TDF FTC & -0.04872 & $\mathrm{~ns}$ \\
\hline TDF vs EVG TDF FTC & 0.06454 & $\mathrm{~ns}$ \\
\hline TDF vs TDF FTC & 0.1616 & \\
\hline TDF vs Blebb & 0.03036 & $\mathrm{~ns}$ \\
\hline FTC vs DTG TDF FTC & -0.06056 & ns \\
\hline FTC vs EVG TDF FTC & 0.05270 & $\mathrm{~ns}$ \\
\hline FTC vs TDF FTC & 0.1497 & $\mathrm{~ns}$ \\
\hline FTC vs Blebb & 0.01852 & $\mathrm{~ns}$ \\
\hline DTG TDF FTC vs EVG TDF FTC & 0.1133 & ns \\
\hline DTG TDF FTC vs TDF FTC & 0.2103 & ** \\
\hline DTG TDF FTC vs Blebb & 0.07909 & ns \\
\hline EVG TDF FTC vs TDF FTC & 0.09705 & $\mathrm{~ns}$ \\
\hline EVG TDF FTC vs Blebb & -0.03418 & $\mathrm{~ns}$ \\
\hline TDF FTC vs Blebb & -0.1312 & ns \\
\hline
\end{tabular}




\section{Percent Active}

\section{One Day Treatment}

\begin{tabular}{|l|r|r|}
\hline Tukey's Multiple & $\begin{array}{l}\text { Mean } \\
\text { Comparison Test }\end{array}$ & Significance \\
\hline DMSO vs DTG & -5.503 & $\mathrm{~ns}$ \\
\hline DMSO vs EVG & 2.569 & $\mathrm{~ns}$ \\
\hline DMSO vs TDF & -7.743 & $\mathrm{~ns}$ \\
\hline DMSO vs FTC & -5.904 & $\mathrm{~ns}$ \\
\hline DMSO vs DTG TDF FTC & -7.779 & $\mathrm{~ns}$ \\
\hline DMSO vs EVG TDF FTC & -5.569 & $\mathrm{~ns}$ \\
\hline DMSO vs TDF FTC & 8.073 & $\mathrm{~ns}$ \\
\hline DTG vs EVG & -2.239 & $\mathrm{~ns}$ \\
\hline DTG vs TDF & -0.4002 & $\mathrm{~ns}$ \\
\hline DTG vs FTC & 23.07 & $\mathrm{~ns}$ \\
\hline DTG vs DTG TDF FTC & -2.276 & $\mathrm{~ns}$ \\
\hline DTG vs EVG TDF FTC & -0.06578 & $\mathrm{~ns}$ \\
\hline DTG vs TDF FTC & -10.31 & $\mathrm{~ns}$ \\
\hline EVG vs TDF & -8.473 & $\mathrm{~ns}$ \\
\hline EVG vs FTC & 15.00 & $\mathrm{~ns}$ \\
\hline EVG vs DTG TDF FTC & -10.35 & $\mathrm{~ns}$ \\
\hline EVG vs EVG TDF FTC & -8.138 & $\mathrm{~ns}$ \\
\hline EVG vs TDF FTC & 1.839 & $\mathrm{~ns}$ \\
\hline TDF vs FTC & 25.31 & $\mathrm{~ns}$ \\
\hline TDF vs DTG TDF FTC & -0.03650 & $\mathrm{~ns}$ \\
\hline TDF vs EVG TDF FTC & 2.174 & $\mathrm{~ns}$ \\
\hline TDF vs TDF FTC & 23.47 & $\mathrm{~ns}$ \\
\hline FTC vs DTG TDF FTC & -1.876 & $\mathrm{~ns}$ \\
\hline FTC vs EVG TDF FTC & 0.3345 & $\mathrm{~ns}$ \\
\hline FTC vs TDF FTC & -25.35 & $\mathrm{~ns}$ \\
\hline DTG TDF FTC vs EVG TDF FTC & -23.14 & 2.210 \\
\hline DTG TDF FTC vs TDF FTC & & $\mathrm{ns}$ \\
\hline EVG TDF FTC vs TDF FTC & & \\
\hline
\end{tabular}

\section{Seven Day Treatment}

\begin{tabular}{|c|c|c|}
\hline $\begin{array}{l}\text { Tukey's Multiple } \\
\text { Comparison Test }\end{array}$ & \begin{tabular}{|l} 
Mean \\
Difference
\end{tabular} & Significance \\
\hline DMSO vs DTG & 20.72 & \\
\hline DMSO vs EVG & 66.99 & $\star * \star$ \\
\hline DMSO vs TDF & 7.533 & $\mathrm{~ns}$ \\
\hline DMSO vs FTC & 0.8424 & $\mathrm{~ns}$ \\
\hline DMSO vs DTG TDF FTC & 53.36 & *** \\
\hline DMSO vs EVG TDF FTC & 71.02 & *** \\
\hline DMSO vs TDF FTC & 18.47 & \\
\hline DTG vs EVG & 46.27 & *** \\
\hline DTG vs TDF & -13.19 & $\mathrm{~ns}$ \\
\hline DTG vs FTC & -19.88 & \\
\hline DTG vs DTG TDF FTC & 32.64 & *** \\
\hline DTG vs EVG TDF FTC & 50.30 & *** \\
\hline DTG vs TDF FTC & -2.247 & $\mathrm{~ns}$ \\
\hline EVG vs TDF & -59.46 & *** \\
\hline EVG vs FTC & -66.15 & *** \\
\hline EVG vs DTG TDF FTC & -13.63 & $\mathrm{~ns}$ \\
\hline EVG vs EVG TDF FTC & 4.024 & $\mathrm{~ns}$ \\
\hline EVG vs TDF FTC & -48.52 & $* * *$ \\
\hline TDF vs FTC & -6.690 & $\mathrm{~ns}$ \\
\hline TDF vs DTG TDF FTC & 45.83 & *** \\
\hline TDF vs EVG TDF FTC & 63.48 & *** \\
\hline TDF vs TDF FTC & 10.94 & $\mathrm{~ns}$ \\
\hline FTC vs DTG TDF FTC & 52.52 & *** \\
\hline FTC vs EVG TDF FTC & 70.17 & *** \\
\hline FTC vs TDF FTC & 17.63 & $\mathrm{~ns}$ \\
\hline DTG TDF FTC vs EVG TDF FTC & 17.66 & ns \\
\hline DTG TDF FTC vs TDF FTC & -34.89 & *** \\
\hline EVG TDF FTC vs TDF FTC & -52.55 & $* * \star$ \\
\hline
\end{tabular}




\section{Event Frequency}

\section{One Day Treatment}

\begin{tabular}{|l|r|r|}
\hline Tukey's Multiple & $\begin{array}{l}\text { Mean } \\
\text { Domparison Test }\end{array}$ & Significance \\
\hline DMSO vs DTG & -0.004991 & $\mathrm{~ns}$ \\
\hline DMSO vs EVG & -0.02025 & $\mathrm{~ns}$ \\
\hline DMSO vs TDF & 0.001156 & $\mathrm{~ns}$ \\
\hline DMSO vs FTC & -0.01612 & $\mathrm{~ns}$ \\
\hline DMSO vs DTG TDF FTC & -0.015913 & $\mathrm{~ns}$ \\
\hline DMSO vs EVG TDF FTC & -0.01128 & $\mathrm{~ns}$ \\
\hline DMSO vs TDF FTC & -0.01526 & $\mathrm{~ns}$ \\
\hline DTG vs EVG & 0.006147 & $\mathrm{~ns}$ \\
\hline DTG vs TDF & -0.01113 & $\mathrm{~ns}$ \\
\hline DTG vs FTC & 0.01060 & $\mathrm{~ns}$ \\
\hline DTG vs DTG TDF FTC & -0.01092 & $\mathrm{~ns}$ \\
\hline DTG vs EVG TDF FTC & -0.006288 & $\mathrm{~ns}$ \\
\hline DTG vs TDF FTC & 0.02141 & $\mathrm{~ns}$ \\
\hline EVG vs TDF & 0.004131 & $\mathrm{~ns}$ \\
\hline EVG vs FTC & 0.02587 & $\mathrm{~ns}$ \\
\hline EVG vs DTG TDF FTC & 0.004345 & $\mathrm{~ns}$ \\
\hline EVG vs EVG TDF FTC & 0.008976 & $\mathrm{~ns}$ \\
\hline EVG vs TDF FTC & -0.01728 & $\mathrm{~ns}$ \\
\hline TDF vs FTC & 0.004456 & $\mathrm{~ns}$ \\
\hline TDF vs DTG TDF FTC & -0.01707 & $\mathrm{~ns}$ \\
\hline TDF vs EVG TDF FTC & -0.01244 & $\mathrm{~ns}$ \\
\hline TDF vs TDF FTC & 0.02174 & $\mathrm{~ns}$ \\
\hline FTC vs DTG TDF FTC & 0.0002133 & $\mathrm{~ns}$ \\
\hline FTC vs EVG TDF FTC & 0.004844 & $\mathrm{~ns}$ \\
\hline FTC vs TDF FTC & -0.02152 & $\mathrm{~ns}$ \\
\hline DTG TDF FTC vs EVG TDF FTC & -0.01689 & $\mathrm{~ns}$ \\
\hline DTG TDF FTC vs TDF FTC & 0.004631 & \\
\hline EVG TDF FTC vs TDF FTC & & \\
\hline
\end{tabular}

\section{Seven Day Treatment}

\begin{tabular}{|c|c|c|}
\hline \begin{tabular}{|l} 
Tukey's Multiple \\
Comparison Test
\end{tabular} & $\begin{array}{l}\text { Mean } \\
\text { Difference }\end{array}$ & Significance \\
\hline DMSO vs DTG & 0.008768 & $\mathrm{~ns}$ \\
\hline DMSO vs EVG & 0.04941 & $* \star$ \\
\hline DMSO vs TDF & -0.001770 & $\mathrm{~ns}$ \\
\hline DMSO vs FTC & -0.004350 & ns \\
\hline DMSO vs DTG TDF FTC & 0.03159 & ns \\
\hline DMSO vs EVG TDF FTC & 0.07615 & *** \\
\hline DMSO vs TDF FTC & 0.003081 & ns \\
\hline DTG vs EVG & 0.04064 & \\
\hline DTG vs TDF & -0.01054 & $\mathrm{~ns}$ \\
\hline DTG vs FTC & -0.01312 & $\mathrm{~ns}$ \\
\hline DTG vs DTG TDF FTC & 0.02282 & ns \\
\hline DTG vs EVG TDF FTC & 0.06738 & $* * \star$ \\
\hline DTG vs TDF FTC & -0.005688 & $\mathrm{~ns}$ \\
\hline EVG vs TDF & -0.05118 & ** \\
\hline EVG vs FTC & -0.05376 & $* * \star$ \\
\hline EVG vs DTG TDF FTC & -0.01782 & $\mathrm{~ns}$ \\
\hline EVG vs EVG TDF FTC & 0.02674 & ns \\
\hline EVG vs TDF FTC & -0.04633 & $* *$ \\
\hline TDF vs FTC & -0.002579 & ns \\
\hline TDF vs DTG TDF FTC & 0.03336 & ns \\
\hline TDF vs EVG TDF FTC & 0.07792 & $* * \star$ \\
\hline TDF vs TDF FTC & 0.004851 & ns \\
\hline FTC vs DTG TDF FTC & 0.03594 & \\
\hline FTC vs EVG TDF FTC & 0.08050 & $* * \star$ \\
\hline FTC vs TDF FTC & 0.007430 & $\mathrm{~ns}$ \\
\hline DTG TDF FTC vs EVG TDF FTC & 0.04456 & ** \\
\hline DTG TDF FTC vs TDF FTC & -0.02851 & $\mathrm{~ns}$ \\
\hline EVG TDF FTC vs TDF FTC & -0.07307 & *** \\
\hline
\end{tabular}




\section{Mean Peak Amplitude}

\section{One Day Treatment}

\begin{tabular}{|l|r|r|}
\hline Tukey's Multiple & $\begin{array}{l}\text { Mean } \\
\text { Comparison Test }\end{array}$ & Significance \\
\hline DMSO vs DTG & -1.099 & $\mathrm{~ns}$ \\
\hline DMSO vs EVG & 0.6888 & $\mathrm{~ns}$ \\
\hline DMSO vs TDF & -4.173 & $\mathrm{~ns}$ \\
\hline DMSO vs FTC & 1.063 & $\mathrm{~ns}$ \\
\hline DMSO vs DTG TDF FTC & -1.797 & $\mathrm{~ns}$ \\
\hline DMSO vs EVG TDF FTC & -1.659 & $\mathrm{~ns}$ \\
\hline DMSO vs TDF FTC & 1.787 & $\mathrm{~ns}$ \\
\hline DTG vs EVG & -3.074 & $\mathrm{~ns}$ \\
\hline DTG vs TDF & 0.03589 & $\mathrm{~ns}$ \\
\hline DTG vs FTC & 3.066 & $\mathrm{~ns}$ \\
\hline DTG vs DTG TDF FTC & -0.6987 & $\mathrm{~ns}$ \\
\hline DTG vs EVG TDF FTC & -0.5604 & $\mathrm{~ns}$ \\
\hline DTG vs TDF FTC & -4.862 & $\mathrm{~ns}$ \\
\hline EVG vs TDF & -1.752 & $\mathrm{~ns}$ \\
\hline EVG vs FTC & 1.279 & $\mathrm{~ns}$ \\
\hline EVG vs DTG TDF FTC & -2.486 & $\mathrm{~ns}$ \\
\hline EVG vs EVG TDF FTC & -2.348 & $\mathrm{~ns}$ \\
\hline EVG vs TDF FTC & 3.110 & $\mathrm{~ns}$ \\
\hline TDF vs FTC & 6.140 & $\mathrm{~ns}$ \\
\hline TDF vs DTG TDF FTC & 2.375 & $\mathrm{~ns}$ \\
\hline TDF vs EVG TDF FTC & 2.514 & $\mathrm{~ns}$ \\
\hline TDF vs TDF FTC & 3.030 & $\mathrm{~ns}$ \\
\hline FTC vs DTG TDF FTC & -0.7346 & $\mathrm{~ns}$ \\
\hline FTC vs EVG TDF FTC & -0.5963 & $\mathrm{~ns}$ \\
\hline FTC vs TDF FTC & -3.765 & -3.627 \\
\hline DTG TDF FTC vs EVG TDF FTC & 0.1383 & $\mathrm{~ns}$ \\
\hline DTG TDF FTC vs TDF FTC & & \\
\hline EVG TDF FTC vs TDF FTC & & \\
\hline
\end{tabular}

\section{Seven Day Treatment}

\begin{tabular}{|c|c|c|}
\hline $\begin{array}{l}\text { Tukey's Multiple } \\
\text { Comparison Test }\end{array}$ & \begin{tabular}{|l} 
Mean \\
Difference
\end{tabular} & Significance \\
\hline DMSO vs DTG & 6.825 & ns \\
\hline DMSO vs EVG & 10.96 & \\
\hline DMSO vs TDF & -4.487 & ns \\
\hline DMSO vs FTC & 1.877 & ns \\
\hline DMSO vs DTG TDF FTC & 6.521 & $\mathrm{~ns}$ \\
\hline DMSO vs EVG TDF FTC & 9.934 & \\
\hline DMSO vs TDF FTC & -2.649 & ns \\
\hline DTG vs EVG & 4.134 & $\mathrm{~ns}$ \\
\hline DTG vs TDF & -11.31 & ** \\
\hline DTG vs FTC & -4.948 & ns \\
\hline DTG vs DTG TDF FTC & -0.3046 & ns \\
\hline DTG vs EVG TDF FTC & 3.109 & ns \\
\hline DTG vs TDF FTC & -9.475 & \\
\hline EVG vs TDF & -15.45 & *** \\
\hline EVG vs FTC & -9.082 & ns \\
\hline EVG vs DTG TDF FTC & -4.439 & ns \\
\hline EVG vs EVG TDF FTC & -1.025 & ns \\
\hline EVG vs TDF FTC & -13.61 & *** \\
\hline TDF vs FTC & 6.364 & ns \\
\hline TDF vs DTG TDF FTC & 11.01 & \\
\hline TDF vs EVG TDF FTC & 14.42 & *** \\
\hline TDF vs TDF FTC & 1.837 & ns \\
\hline FTC vs DTG TDF FTC & 4.643 & ns \\
\hline FTC vs EVG TDF FTC & 8.056 & ns \\
\hline FTC vs TDF FTC & -4.527 & ns \\
\hline DTG TDF FTC vs EVG TDF FTC & 3.413 & ns \\
\hline DTG TDF FTC vs TDF FTC & -9.170 & ns \\
\hline EVG TDF FTC vs TDF FTC & -12.58 & ** \\
\hline
\end{tabular}

